\title{
Spark Plug Simulation with the Use of Three Types of Fuels in Direct Injection Engines for the Evaluation of Polluting Factors
}

\section{Simulación de las Bujías con el Uso de Tres Tipos de Combustibles en Motores de Inyec- ción Directa para la Evaluación de Factores Contaminantes}

VII International Congress of

Science, Technology,

Entrepreneurship and

Innovation (SECTEI 2020)

Corresponding Author:

E. Jimenez

e_jimenez28@hotmail.com

Published: 26 August 2021

Production and Hosting by

Knowledge E

(c) E. Jimenez et al. This article is distributed under the terms of the Creative Commons Attribution License, which permits unrestricted use and redistribution provided that the original author and source are credited.
S OPEN ACCESS

\author{
E. Jimenez ${ }^{1}$, R. Molina ${ }^{2}$, and V. Andrade ${ }^{2}$ \\ ${ }^{1}$ Ministerio de Educación \\ 2I.T.Q.
}

\section{Abstract}

The present work has as main objective the use of a biofuel (Ecopaís) in a direct injection vehicle, it is an option to reduce damage to health and the environment, for this a static thermal simulation will be done in the spark plug, to compare the results of the aforementioned software using On Board tests, in a $1500 \mathrm{cc}$ engine. The measurements of the emission factors of $\mathrm{CO}$, $\mathrm{HC}$ and NOx gases will be considered in a route established in the city of Quito from 2399 to 2870 meters above sea level. The interaction of the element is carried out in the ANSYS Academic program which is 14977 nodes and 7523 elements to be studied with automatic meshing, obtaining that the Ecopaís and Ecopaís + Ferox fuels have the highest heat flow with a $5 \%$ divergence compared to the Extra fuel + Ferox. There is a significant reduction in pollutant emissions of 3\% of CO with the use of Ecopaís in comparison to Extra + Ferox fuel, in the case of HC, Ecopaís and Ecopaís + Ferox fuels with 3\% lower emissions compared to Extra fuel + Ferox, and in NOx, fuels that have Extra + Ferox and Ecopaís + Ferox solid additives are 3 and 3.5\% lower compared to Ecopaís fuel, respectively.

Keywords: biofuel, termal, on board, ferox, emission factors.

\section{Resumen}

El presente trabajo tiene como objetivo fundamental la utilización de un biocombustible (Ecopaís) en un vehículo de inyección directa, es una opción para disminuir daños a la salud y al medio ambiente, para ello se hará una simulación térmica estática en la bujía de encendido, para comparar los resultados del mencionado software mediante pruebas On Board, en un motor de $1500 \mathrm{cc}$. Las mediciones de los factores de emisión de gases de CO, HC y NOx, se contemplará en una ruta establecida en la ciudad de Quito de 2399 hasta 2870 m.s.n.m. La interacción del elemento se realiza en el programa ANSYS Academic que es de 14977 nodos y 7523 elementos a estudiar con el mallado automático, obteniendo que los combustibles Ecopaís y Ecopaís+Ferox tienen el mayor flujo de calor con una divergencia del $5 \%$ en comparación del combustible Extra + Ferox. Se evidencia una reducción significativa de emisiones contaminantes del $2.5 \%$ del $\mathrm{CO}$ con el uso del Ecopaís en comparación del combustible Extra + Ferox, en el caso de HC los combustibles Ecopaís y Ecopaís + Ferox con un $1 \%$ menor en emisiones en comparación al combustible Extra + Ferox, y en el NOx los combustibles que tienen aditivo sólido Extra+Ferox y Ecopaís+Ferox son menores en un 6 y $4 \%$ con respecto al combustible Ecopaís respectivamente. 


\section{Introducción}

La elevada contaminación ambiental en el Ecuador lleva a realizar estudios de factibilidad del uso de nuevos combustibles que generen prestaciones similares y reduzcan las emisiones contaminantes producidas por los automotores que actualmente trabajan con gasolina. Hoy en día existe un aumento de temperatura global de $2^{\circ} \mathrm{C}$ debido a la contaminación medioambiental [1].

El biocombustible Ecopaís está compuesto con un 5\% de etanol proveniente de la caña de azúcar y 95\% de gasolina extra [2]. El alcohol que contiene esta combustible permite mantener limpio el motor. Además, el consumo de este biocombustible no sólo reduce las emisiones de gases contaminantes al ambiente, sino que impulsa el desarrollo de la agroindustria nacional [3].

ECOPAÍS tiene el mismo octanaje (87 octanos) y precio del combustible Extra. Para la producción de gasolina Extra con 87 octanos se requiere mezclar un 76\% de NAO (nafta de alto octano), las cuales se importan, con $24 \%$ de naftas de bajo octano (NBO); mientras que para la producción de ECOPAís, con la misma cantidad de octanos, se requiere $62 \%$ de NAO, $33 \%$ de NBO y un $5 \%$ de bioetanol.

De igual forma el uso de aditivos ayuda a problemas del motor, tales como consumo excesivo de combustible, exceso de emisiones dañinas de escape y altos costos de mantenimiento. Los problemas en el combustible y la combustión incompleta causan la falla completa del motor [4].

La temperatura de los gases de escape es un parámetro muy importante de determinar, ya que una temperatura arriba de los valores comunes (entre $400^{\circ} \mathrm{C}$ y $600^{\circ} \mathrm{C}$ ) puede significar un alto contenido de NOx en los gases, ya que éstos se forman durante la combustión a temperaturas arriba de $2,000^{\circ} \mathrm{C}$, por lo tanto, es de esperarse que los gases de escape salgan a una temperatura mayor [5].

En la Figura 1, la chispa de encendido debe saltar con cierta antelación con respecto al PMS para que la presión de combustión alcance su valor máximo, poco después de que el pistón inicie la carrera descendente una vez superado dicho PMS. Este punto óptimo depende de las características constructivas del motor (tamaño de biela, diámetro del pistón, tamaño de la muñequilla del cigüeñal, etc.) [6].

Por lo mencionado anteriormente, el trabajo tiene como objetivo: Evaluar y simular la bujía de acuerdo con el uso del efecto del etanol y aditivo en el motor de combustión interna, mediante una validación experimental. Y con este tipo de conocimiento de particular relevancia permitirá realizar una definición informada de las políticas y regulaciones públicas que se implementarán en el futuro con respecto a emisiones de gases contaminantes, para lograr un medio ambiente urbano más limpio y saludable. 


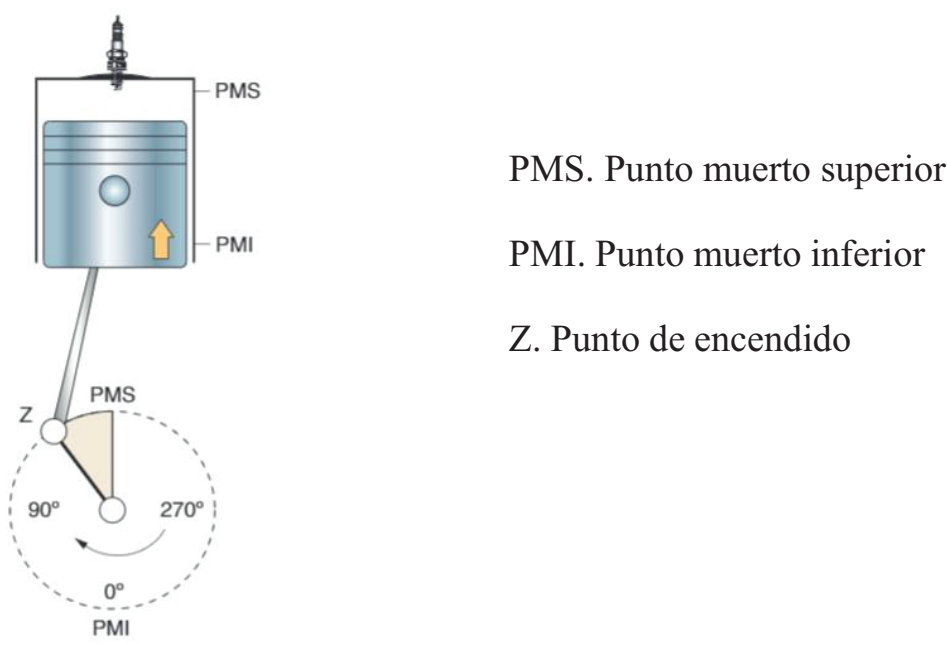

Figure 1

Punto de encendido de acuerdo con la posición del cigüeñal [6].

\section{Metodología}

\subsection{Vehículo de prueba}

Se optó por un vehículo de la marca KIA modelo Rio Stylus, ya que es una de las marcas mejor posesionadas en la venta de vehículos en todo el país, el kilometraje es de 91.157 $\mathrm{km}$, tomando en cuenta que para las pruebas que se van a desarrollar en este vehículo las condiciones del motor, dirección y transmisión deben tener un mínimo deterioro en sus elementos.

Por lo general se deben utilizar vehículos con tecnología Euro III y IV, ya que significativamente las emisiones de gases contaminantes son bajas con vehículos respecto a Euro I [7].

La mayoría de estos estudios utilizan procedimientos de medición que son costosos y consumen tiempo, y por lo tanto, se tiende a centrarse en un solo parámetro o una pequeña muestra de vehículos [8].

Adicionalmente, se toma en cuenta que las emisiones de este tipo de vehículo se verifican en ciclos europeos de acuerdo con la norma NTE INEN 2204:2002.

En la Tabla 1 se encuentra los parámetros de la cámara de combustión, diámetro del pistón, carrera y relación de compresión del vehículo KIA R STYLUS.

\subsection{Tipos de combustible}

Para el estudio se toma tres propuestas de combustibles, una de uso convencional Ecopaís, y dos mezclas con el aditivo Ferox, utilizando $75 \mathrm{~L}$ de combustible y una pastilla 


\section{Table 1}

Parámetros de la cámara de cilindro del vehículo KIA R STYLUS.

Parámetros
Diámetro del Pistón
Carrera
Relación de Compresión
Calidad de Combustible
Torque
Potencia
Angulo de Chispa
Duración de la Chispa
Distancia Horizontal de la bujía
Revoluciones del motor

Magnitud
$75,5 \mathrm{~mm}$
$75 \mathrm{~mm}$
$9,5: 1$
RON 87
$125,4 \mathrm{Nm}$
$97 \mathrm{HP}$
$345^{\circ}$
$8^{\circ}$
$43,9 \mathrm{~mm}$
$6000 \mathrm{rpm}$

Nota: Resumen de gases contaminantes [9].

de este aditivo de $1 \mathrm{~g}$, en la Tabla 2 se muestra los combustibles con la cantidad del aditivo y sus densidades.

\section{Table 2}

Tipos de combustible a utilizar.

Tipo de combustible
Ecopaís
Extra + Ferox
Ecopaís + Ferox

Características
Gasolina convencional adicionada
$5 \%$ de etanol
Gasolina Convencional + $0.5 \mathrm{~g}$ de
Ferox
Gasolina convencional adicionada
$5 \%$ de etanol + $0.5 \mathrm{~g}$ de Ferox

Densidad $\left(\mathbf{K g} / \mathbf{m}^{3}\right)$
791,5
620,2
643,8

\subsection{Tipo de ruta}

Para la medición de factores contaminantes se escogió una ruta por diferentes parámetros como: Distancia de recorrido, tráfico, sector (urbano o rural) y situación geográfica, con referencia a esto se inicia desde el SUPERMAXI de Cumbayá (cerca del redondel de la Universidad San Francisco) para después continuar a través de la Av. Interoceánica, Libertador Simón Bolívar, De los Granados, Eloy Alfaro, Río Coca, Shyris, 6 de Diciembre, Francisco de Orellana, Coruña, 12 de Octubre y finaliza en la Universidad Católica (intersección Av. 12 de Octubre y Jorge Washington); recorriendo una distancia aproximada de 9990 m de parte Sub-urbana y 8100 m de parte urbana, con una distancia total de recorrido de $18.090 \mathrm{~m}$. En la Figura 2 se puede apreciar la ruta mencionada. 


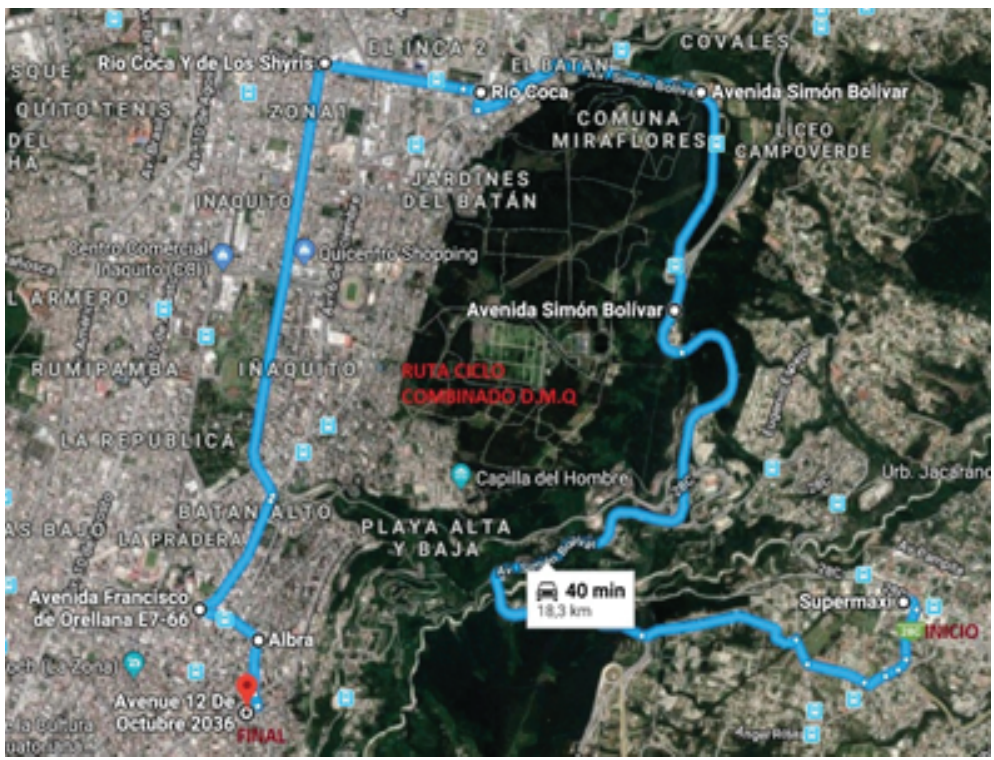

Figure 2

Ruta On Board. Fuente: Referencia [10].

En la Figura 3 se puede apreciar con el software Google Earth, la longitud de la ruta de la siguiente manera: El $47 \%$ del total del recorrido es plano o con pendientes menores a grado 3 , el $10 \%$ contiene pendientes de grado 4 , el $26 \%$ contiene pendientes de grado 6 , el $12 \%$ contiene pendientes de grado -4 , el $5 \%$ contiene pendientes de grado $-6[11]$.

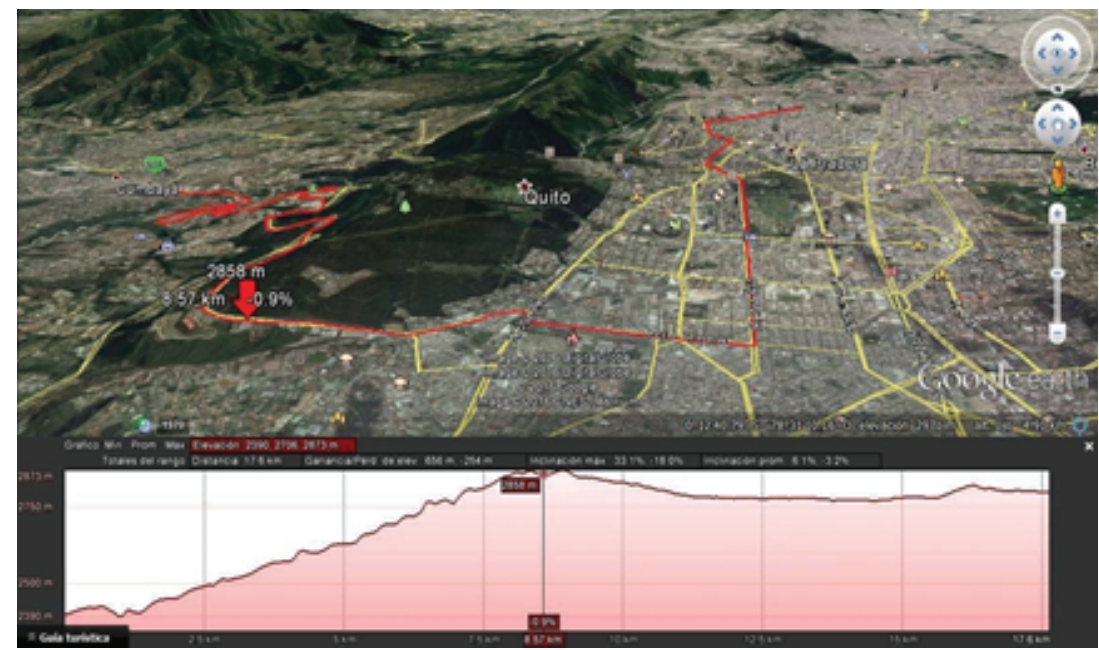

Figure 3

Perfil de elevación de la ruta On Board. Fuente: Referencia [10].

Se puede establecer que el punto más prominente está ubicado en la Av. Libertador Simón Bolívar (altura del Camposanto Monteolivo, $2870 \mathrm{~m}$ ), mientras que el punto de menor altura está en la Av. Interoceánica (altura del Supermaxi de Cumbayá, 2399 m; 
ya que está ubicado en el inicio de la trayectoria y su poca elevación es debido a que se encuentra al ingreso del Valle de Tumbaco [11].

Se determina medir el consumo másico de combustible por el método de la estequiometría, es decir, conociendo el caudal de aire de admisión en $\mathrm{kg} / \mathrm{hr}$ y el valor de la relación aire/combustible (A/F), donde se conoce el valor real del consumo másico de combustible en $\mathrm{kg} / \mathrm{hr}$.

\subsection{Pruebas On Board}

Para el actual análisis se plantea ejecutar pruebas ON BOARD, la prueba de medición a bordo es una cercanía deseable de la cuantificación en lo que respecta a emisiones vehiculares en circunstancias reales. El propósito de este estudio es conseguir la masa real de cada contaminante en función del tiempo, distancia de recorrido y consumo de combustible. Para su siguiente análisis en relación con cada carburante utilizado [12].

El protocolo de pruebas ON BOARD, se encuentra normalizadas en laboratorio por NTE INEN, entre los cuales se indica los equipos a manejar en el proceso de medida de emisiones contaminantes en ruta, anteriormente establecida [13].

La característica primordial de esta prueba radica en tiempo, ya que los resultados se obtienen en un lapso real y en las condiciones reales de operación del automóvil, de este modo se aprecia los factores de contaminación de forma más precisa y en recorridos determinados. La principal desventaja o inconveniente de esta prueba, se realiza al instante de su instalación, ya que se necesita un gran espacio en el habitáculo del automóvil, mayor tiempo para su puesta en marcha y disponibilidad de los diferentes vehículos para cada ensayo o prueba.

Este prototipo de pruebas de experimentación ON BOARD, se utiliza principalmente en tener valores reales de emisiones contaminantes en relación con: $\mathrm{CO}, \mathrm{HC}$ y NOx en circunstancias reales de operación, clima, temperatura ambiente, velocidad y tráfico.

En la Figura 4 se observa cómo van acoplados los componentes para la medición de gases de escape dinámica y consumo de combustible en el automóvil experimental.

A continuación, se encuentra detallado los pasos para la conexión del equipo y mediciones que se realiza en el automóvil KIA R STYLUS

El automóvil se encontrará apagado para la instalación de los equipos, ya que el analizador de gases On Board debe estar seguro para una lectura óptima de los datos de factores de contaminación, como se muestra en la Figura 5.

La instalación en el sistema de escape se debe realizar con cuidado, se debe comprobar que las mangueras estén limpias y bien sujetas al tubo de escape, ya que en el dispositivo On Board los datos a obtener sean lo más precisos a la hora de realizar dichas pruebas, como se aprecia en la Figura 6. 


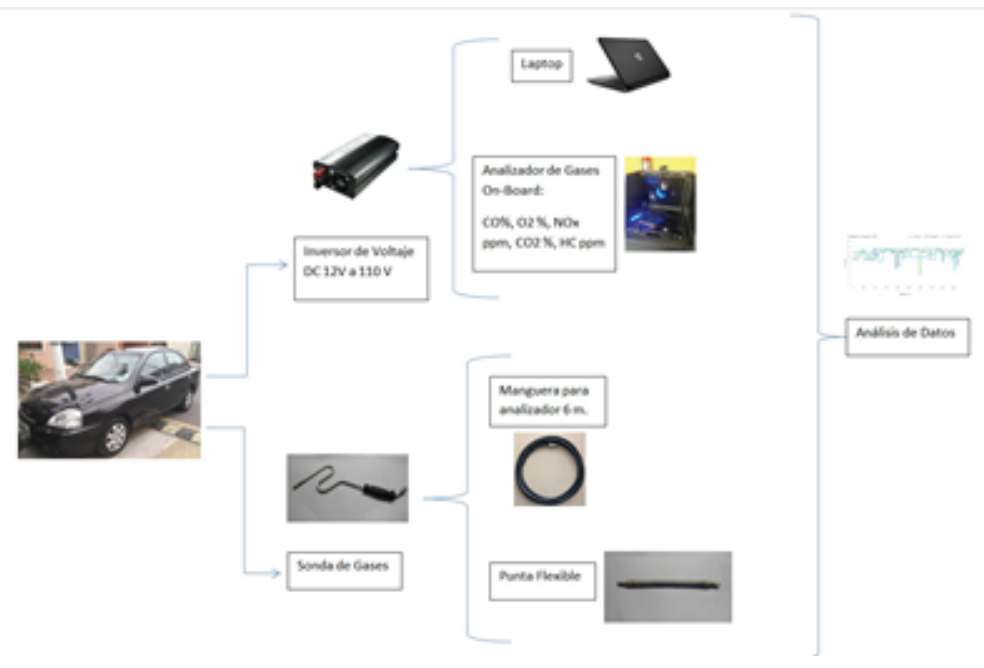

Figure 4

Conexión de equipo On Board en el vehículo.

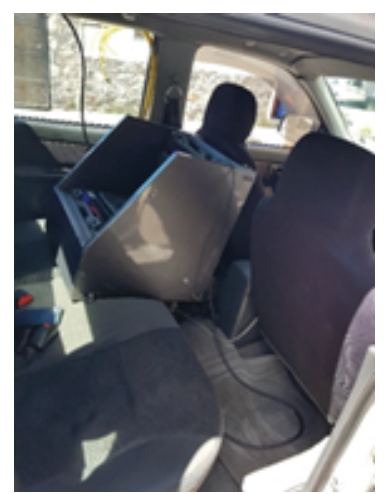

Figure 5

Dispositivo On Board en el vehículo.

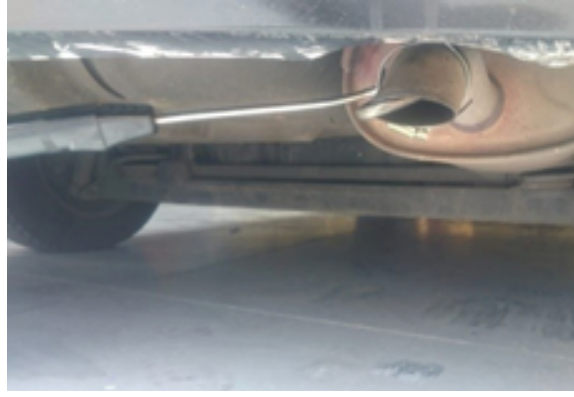

Figure 6

Inspección visual del sistema de escape.

Se procede a retirar el relé de la bomba de combustible para instalar el tanque de combustible externo, y luego utilizar los combustibles que permitirán hacer las pruebas de consumo y emisiones de gases. La presión estimada es de 50 PSI que se 
proporciona manualmente, bombeando el combustible al riel de inyectores, para todo el trayecto y tiempo que demore la ruta. En la Figura 7, se aprecia el tanque externo de combustible.

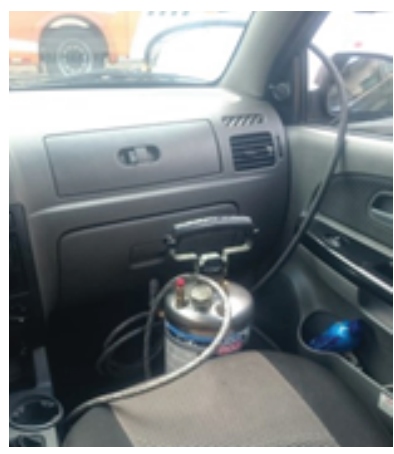

Figure 7

Tanque de combustible instalado en el vehículo.

El aditivo sólido FEROX se combina con los combustibles EXTRA y ECOPAÍs; se debe dejar que se diluya totalmente la pastilla. En las Figura 8 se muestra el aditivo FEROX para los combustibles a comparar.

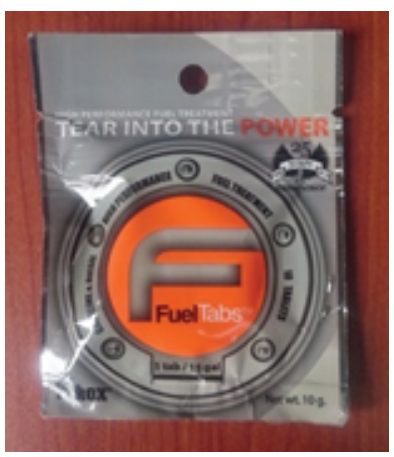

\section{Figure 8}

Pastilla ferox.

Antes de seguir la ruta calibrar el equipo, para este proceso se deja en ralentí el automóvil unos cinco minutos aproximadamente para la calibración automática del equipo, los datos variarán de acuerdo con la forma de conducir, tráfico, clima, y otro tipo de circunstancias.

Al terminar cada recorrido, el restante de combustible es enviado desde el tanque externo a una probeta, comparar los valores iniciales y finales, como se pueden observar en la Figura 9, para obtener el consumo de combustible en cada prueba. Se deben registrar los datos en un dispositivo de almacenamiento, que arroja el equipo On Board, para obtener los valores de emisiones contaminantes y repetir el procedimiento para cada prueba de combustible. 


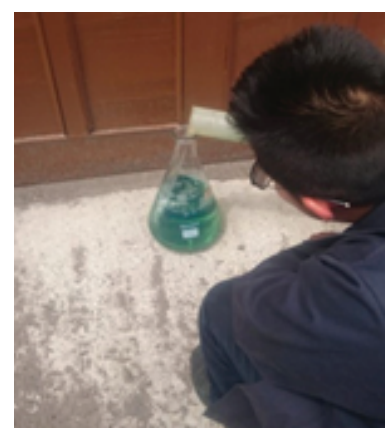

\section{Figure 9}

Medición de combustible en probetas.

\subsection{Simulación de la bujía de encendido en ANSYS}

Con respecto a la simulación de la bujía se realiza en el software ANSYS con las tres propuestas de combustibles, con las temperaturas de $1040^{\circ} \mathrm{C}, 1200^{\circ} \mathrm{C}$ y $1210^{\circ} \mathrm{C}$ de los combustibles Extra + Ferox, Ecopaís y Ecopaís + Ferox respectivamente, obteniendo el análisis térmico estático y flujo de calor; se muestra el desarrollo de un modelo predictivo de la combustión de mezclas de gas natural para motores de encendido por chispa, este modelo se basa en el de giro del cigüeñal (ángulo) para el cálculo de las temperaturas y presión en la cámara de combustión [14]. Se tomarán en cuenta las propiedades térmicas del cobre y acero inoxidables correspondientes a los electrodos negativo y positivo en una bujía (Tablas 3 y 4).

\section{Table 3}

Propiedades rérmicas del electrodo positivo.

Propiedades térmicas del cobre
Calor Específico a $25^{\circ} \mathrm{C}\left(\mathrm{J} \mathrm{K}^{-1} \mathrm{~kg}^{-1}\right)$
Calor Latente de Evaporación $\left(\mathrm{J} \mathrm{g}^{-1}\right)$
Calor Latente de Fusión $\left(\mathrm{J} \mathrm{g}^{-1}\right)$
Coeficiente de Expansión Térmica @0-100C $\left(\times 10^{-6} \mathrm{~K}^{-1}\right)$
Conductividad Térmica a 0-100C $\left(\mathrm{W} \mathrm{m}^{-1} \mathrm{~K}^{-1}\right)$

\section{Valores}

385

4796

205

17,0

401

Table 4

Propiedades térmicas del electrodo negativo.

Propiedades térmicas del acero AISI410
Coeficiente de Expansión Térmica @20-100C (x10-6 $\left.\mathrm{K}^{-1}\right)$
Conductividad Térmica a $23 \mathrm{C}\left(\mathrm{W} \mathrm{m}^{-1} \mathrm{~K}^{-1}\right)$
Temperatura Máxima de Utilización al Aire (C)


Parte del estudio de la bujía se presenta el análisis térmico del elemento ensamblado en el software ANSYS, con los siguientes pasos a realizar:

Antes del post-procesamiento de la bujía, abrir el programa ANSYS, para seleccionar análisis térmico estático, como se encuentra en la Figura 10.

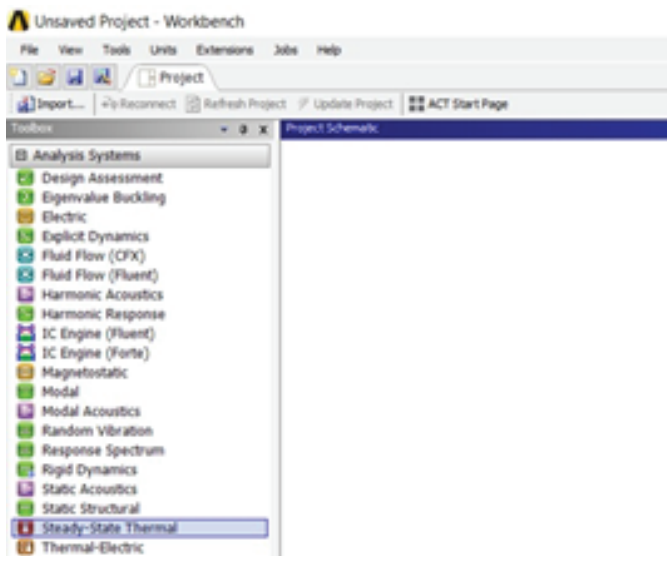

\section{Figure 10}

Software ANSYS opción análisis térmico estático.

Para adicionar los materiales, para este estudio se debe escoger el material cobre para el electrodo, acero al carbono para el roscado, la tuerca y cerámica para el cuerpo de la bujía de encendido como en la Figura 11.

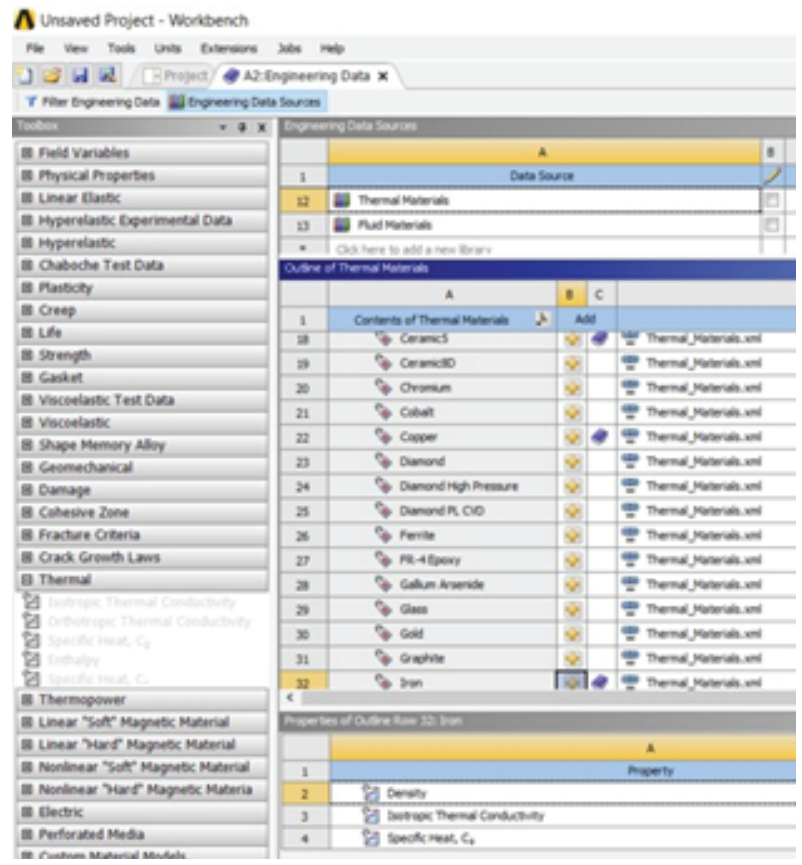

Figure 11

Opción engineering data. 
Dirigirse a geometría, para importar el archivo CAD en formato (igs) de la pieza antes mencionada, dibujada en NX 11, debe estar en ese formato para que pueda ser reconocido por ANSYS, como se observa en la Figura 12.

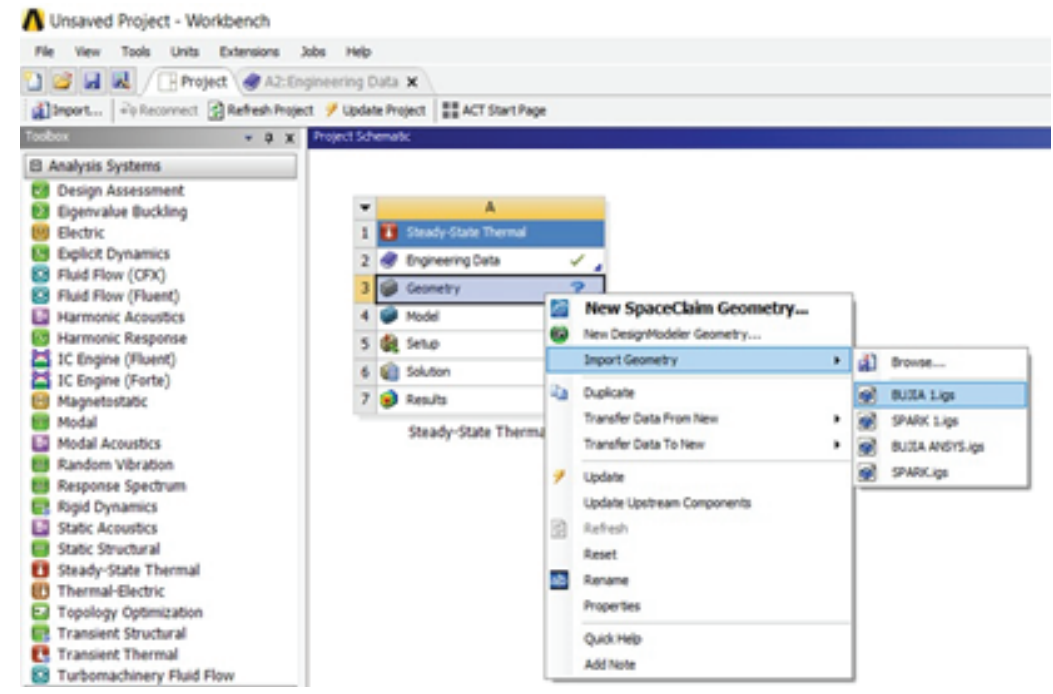

Figure 12

Opciones geometry, import geometry y bujía 1 (igs).

Seleccionar Model, clic derecho y escoger editar, como en la Figura 13. La opción permite colocar las cargas, temperaturas, conexiones, mallado, entre otras, que la pieza dibujada será posteriormente sometida.

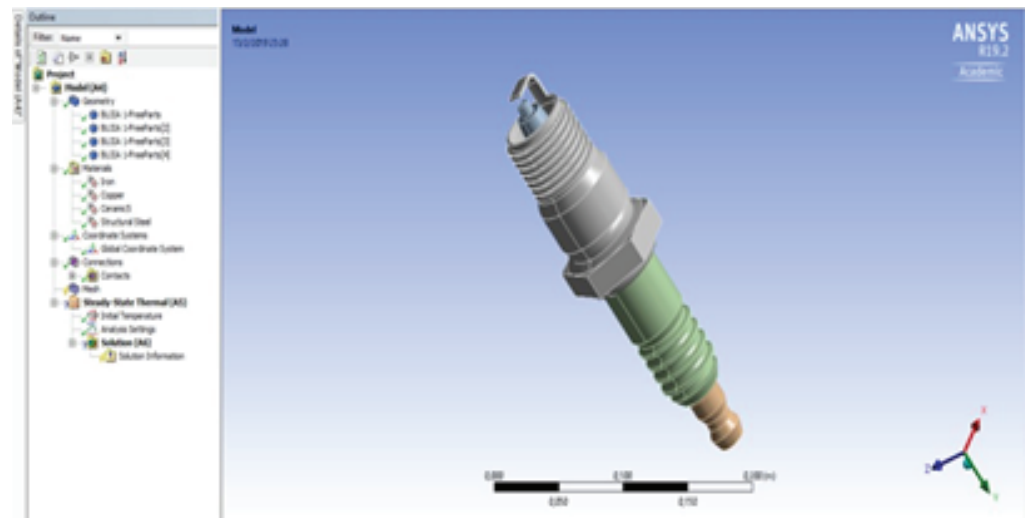

\section{Figure 13}

CAD de la bujía en ANSYS.

Seleccionar el elemento de la pieza para ingresar el tipo de material, en este caso escogemos el material Acero AISI 410; realizar el mismo procedimiento para los demás elementos de la bujía, indicada en las Figura 14.

A continuación, se genera dos tipos de mallado debido a que el cuerpo de la bujía es irregular, el primero es estructurado (hexaédrico) donde va conectado el cable de bujías y el segundo es un mallado híbrido (tetraédrico y hexaédrico) con mayor cantidad de 


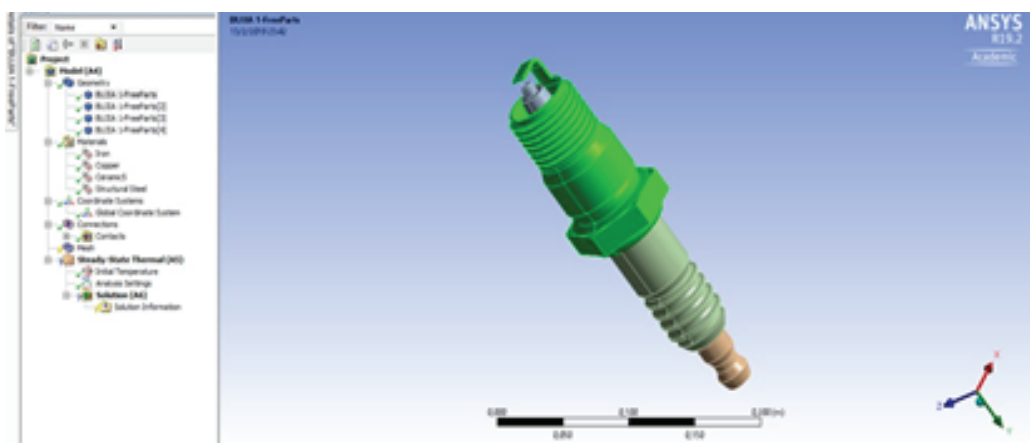

Figure 14

Ingreso de materiales de la bujía.

nodos en el electrodo positivo y negativo de la bujía, ya que en el análisis de resultados arrojará valores más semejantes a la realidad para ser posteriormente comparados, y conocer con cuál combustible existe una mejor combustión y menor emisión de gases, como se observan en las Figuras 15 y 16, donde el proceso de refinación de mallado es de 2,3982 e-004 m, con un total de 14876 nodos y 7464 en elementos a estudiar.

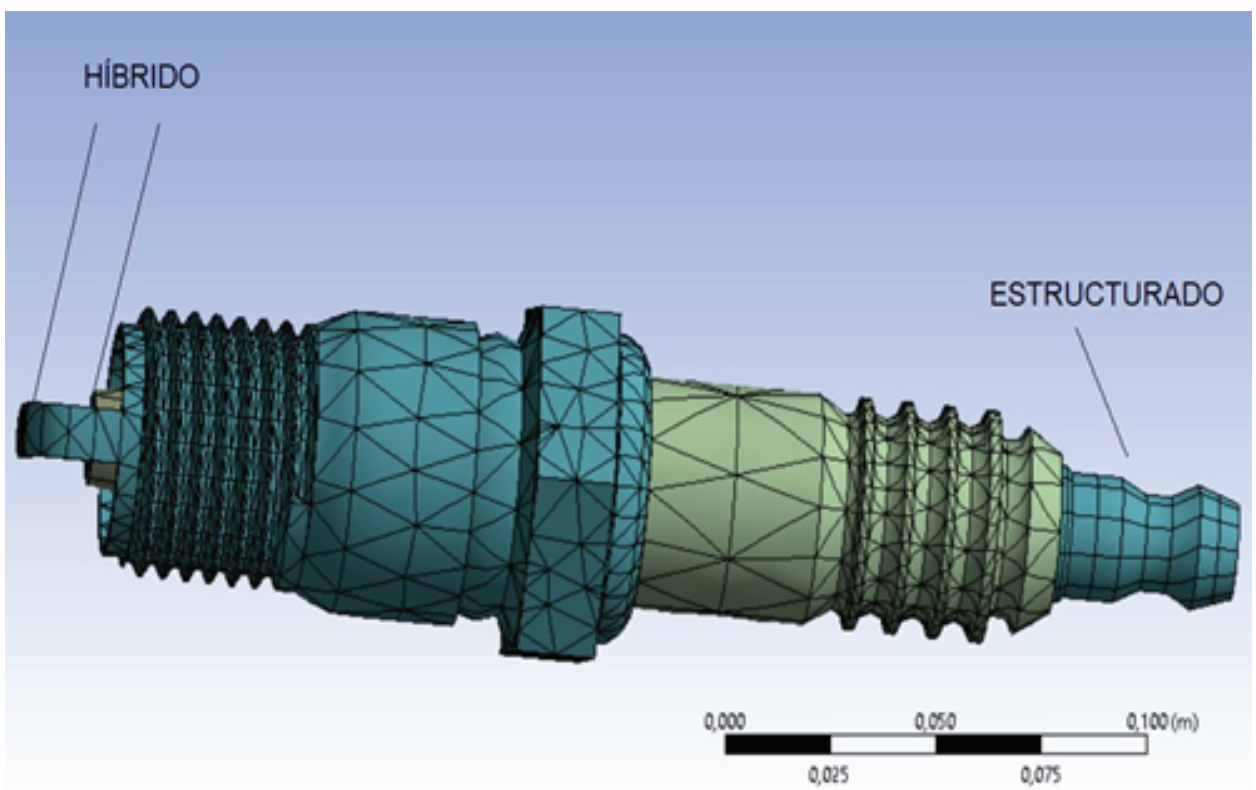

Figure 15

Mallado estructurado e hibrido.

Posteriormente al obtener el mallado, se coloca las cargas térmicas. Se inserta la máxima temperatura que es bordea los $1000^{\circ} \mathrm{C}$ y la mínima que es cerca de los $110^{\circ} \mathrm{C}$ y seleccionar el componente donde se ejecutar la máxima carga de temperatura, como se aprecia en la Figura 17.

Una vez puesta la condición de temperatura ingresar el coeficiente de convicción térmico que es dado por el uso de combustibles para el caso del extra el coeficiente 


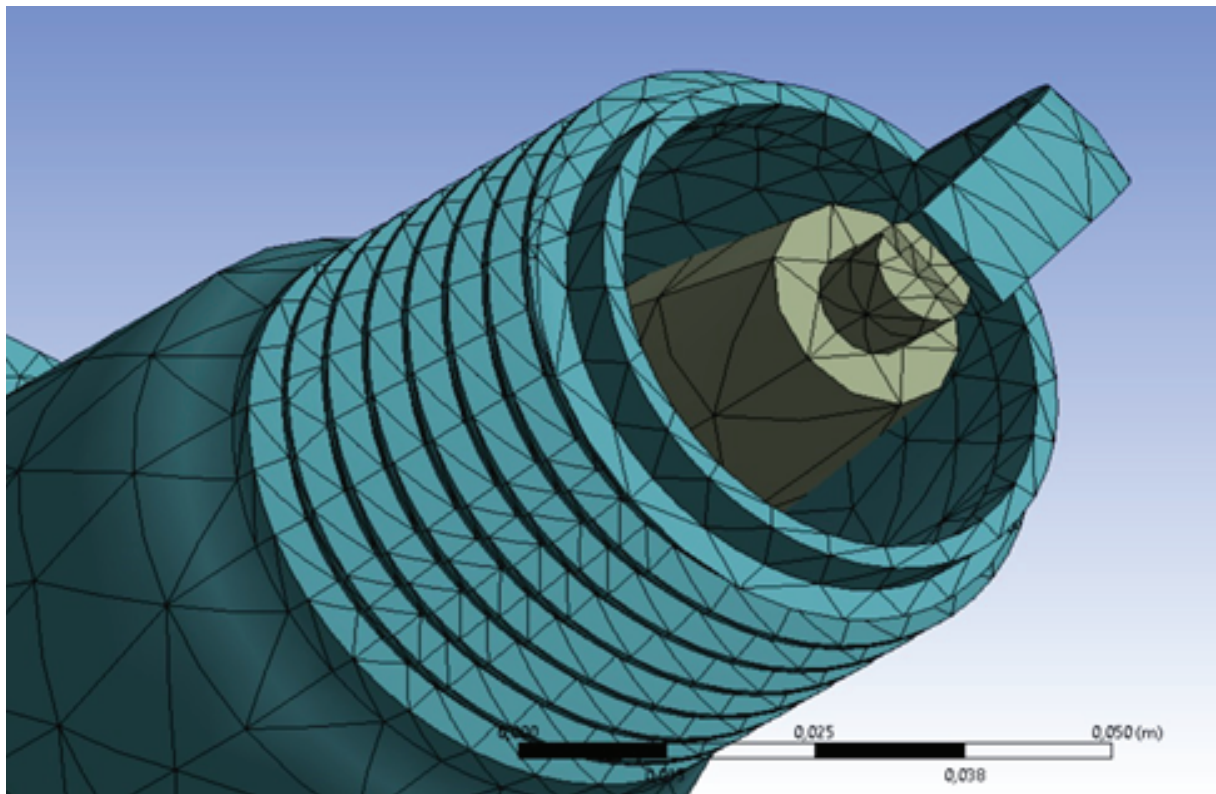

Figure 16

Mallado hibrido electrodo positivo y negativo.

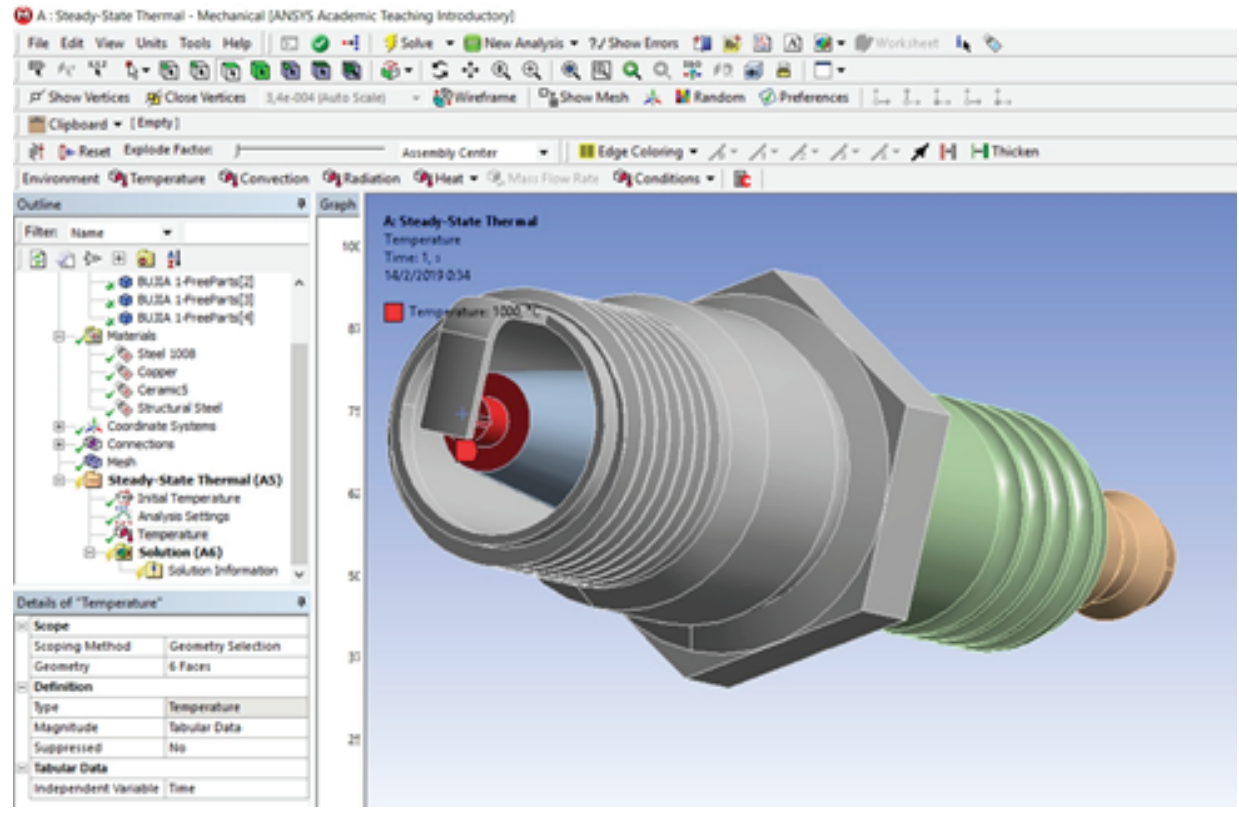

Figure 17

Temperatura en el electrodo.

es de $1800 \mathrm{w} / \mathrm{m} 2^{\circ} \mathrm{C}$ y la temperatura ambiente que es $110^{\circ} \mathrm{C}$, como se observa en las Figura 18.

Se realiza la solución mediante la opción Solve. Esperar el resultado del análisis térmico en la bujía para el proceso de combustión. Finalmente se obtiene los resultados del análisis térmico estático, como se aprecia en la Figura 19. 


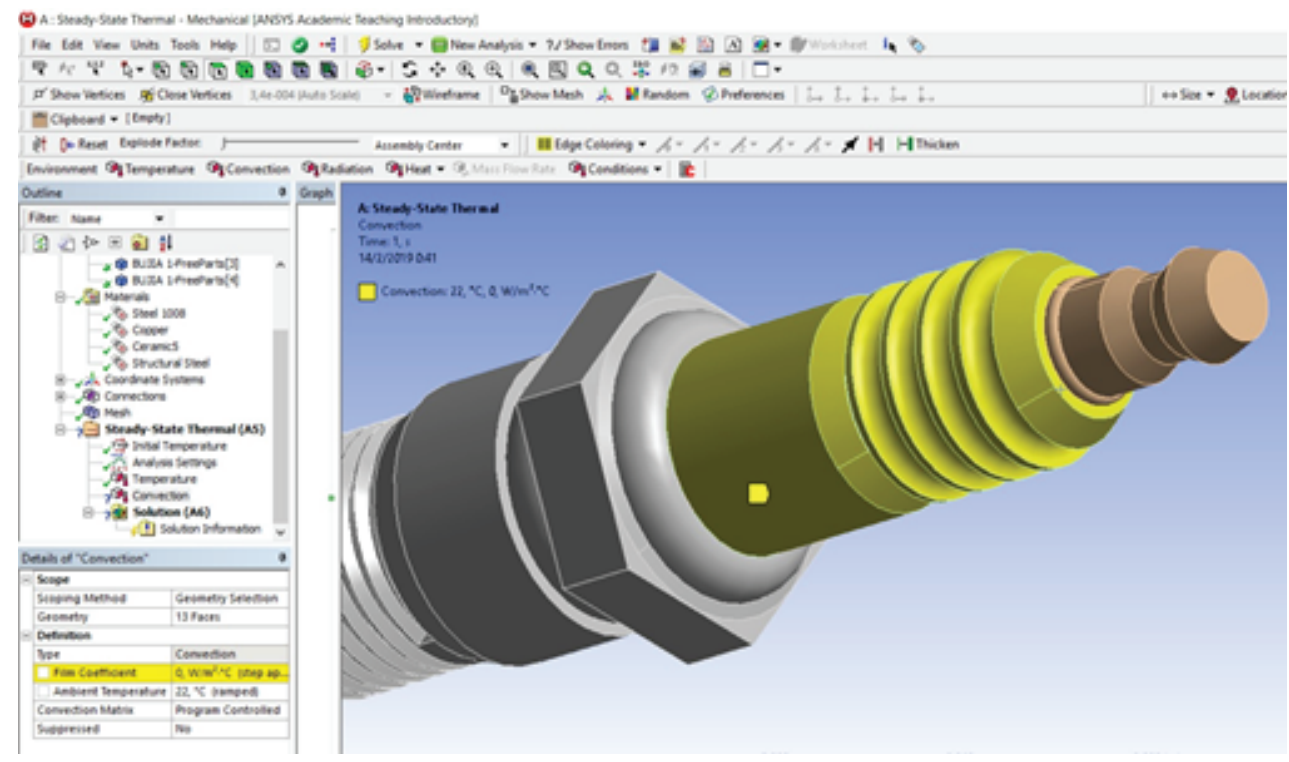

Figure 18

Ingreso de dato de convección térmica.

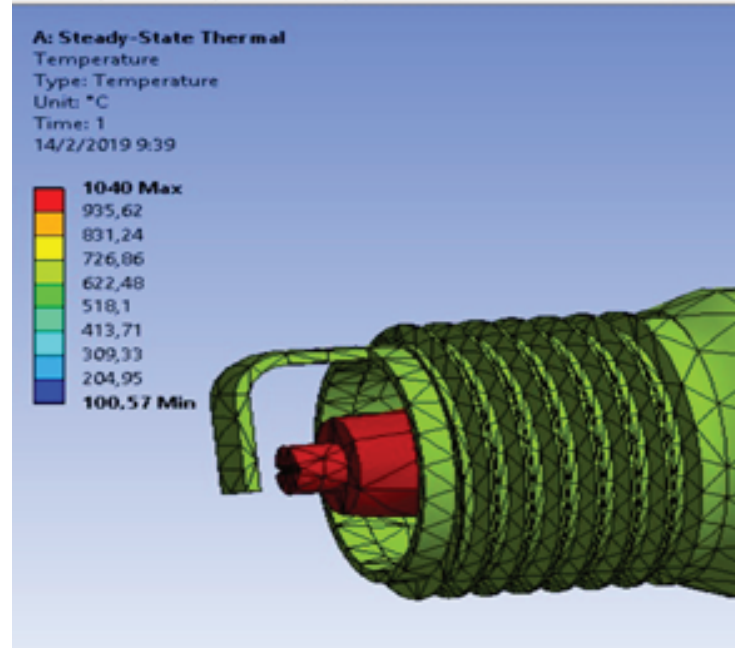

Figure 19

Análisis térmico de la bujía.

\subsection{Cálculo de las factores de emisión}

\subsubsection{Fundamento teórico para el cálculo de los factores de emisión}

Un factor de emisión es una relación entre la cantidad de contaminante emitido a la atmósfera y una unidad de actividad.

Con el conocimiento del peso molecular y la densidad del combustible utilizado es posible calcular la cantidad de $\mathrm{CO}, \mathrm{HC}$ y $\mathrm{NO}$ emitidos por galón de combustible quemado. Sin embargo, la mayoría de los inventarios de emisiones se presenta en 
gramos de contaminante por kilómetro recorrido, para lo cual es necesario introducir el consumo de combustible promedio del vehículo de prueba [15].

En la Tabla 5 se aprecia el peso molecular para un combustible con $80 \%$ peso de carbono y $20 \%$ peso hidrogeno, para obtener una formula $\mathrm{CH} 3$.

\section{Table 5}

Fórmula molecular equivalente $\mathrm{CH} 3$.

\begin{tabular}{|c|c|c|c|c|}
\hline Componente & $\begin{array}{l}\text { Lb por } 100 \mathrm{lb} \text { de } \\
\text { combustible }\end{array}$ & Peso molecular & $\begin{array}{l}\text { Lb mol por } 100 \mathrm{lb} \text { de } \\
\text { combustible }\end{array}$ & $\begin{array}{l}\text { Lb mol por lb mol de } \\
\text { carbono }\end{array}$ \\
\hline Carbono & 80,0 & 12,0 & 6,7 & 1,0 \\
\hline Hidrogeno & 20,0 & 1,0 & 20,0 & 3,0 \\
\hline
\end{tabular}

Nota: Fórmula molecular equivalente $\mathrm{CH} 3$, con un combustible con $80 \%$ de peso de carbono y $20 \%$ de peso de hidrogeno [16].

Para este estudio la formula molecular equivalente $\mathrm{CH}$, va relacionado con el peso molecular del combustible [17].

$$
y=\left(\frac{\% \text { peso } H}{\text { \%peso } C}\right)\left(\frac{M W_{C}}{M W_{H}}\right),
$$

dónde: MWC: Peso molecular del carbono; MWH: Peso molecular del hidrógeno.

En la ecuación 2, se presenta los productos de combustión con mayor repercusión ambiental. Para tal se considera $\mathrm{CO}, \mathrm{H} 2 \mathrm{O}, \mathrm{C} 3 \mathrm{H} 6, \mathrm{CO} 2, \mathrm{~N} 2$ y $\mathrm{NO}$ que tienen gran importancia en el balance de la masa para la combustión [18].

$$
\mathrm{CH}_{Y}+m\left(0.21 \mathrm{O}_{2}+0.79 \mathrm{~N}_{2}\right) \rightarrow a \mathrm{CO}+b \mathrm{H}_{2}+c \mathrm{C}_{3} \mathrm{H}_{6:}+d \mathrm{CO}_{2}+e \mathrm{~N}_{2}+f \mathrm{NO},
$$

donde las variables $\boldsymbol{a}, \boldsymbol{b}, \boldsymbol{c}, \boldsymbol{d}, \boldsymbol{e}, \boldsymbol{f}$ y $\boldsymbol{m}$ son coeficiente estequiométricos s desconocidos y definidos según Caiza y Portilla [17], donde: $m$ : Moles de aire consumido por mol de combustible consumido; $a$ : Moles formados de $\mathrm{CO}$ por mol de combustible consumido; $b$ : moles formados de $\mathrm{H} 2 \mathrm{O}$ por mol de combustible consumido; $c$ : moles formados de $\mathrm{C} 3 \mathrm{H} 6$ por mol de combustible consumido; $d$ : moles formados de $\mathrm{CO} 2$ por mol de combustible consumido; e: moles formadas de N2 por mol de combustible consumido; $f$ : moles formados de NO por mol de combustible consumido.

Los átomos de combustión no pueden ser creados ni destruidos, es tal que la presentación del balance de la masa se describe como:

Elemento Recatarte Productos carbono

$$
1=a+3 c+d,
$$

Elemento Recatarte Productos hidrógeno

$$
y=2 b+6 c,
$$

Elemento Recatarte Productos oxigeno

$$
0.42 m=a+b+2 d+f,
$$


Elemento Recatarte Productos nitrógeno

$$
1.58 m=2 e+f .
$$

Al ser el $\mathrm{CO} 2$ el gas predominante, en los residuos de combustión se pretende relacionar este gas con el resto de los residuos que son indispensables en la relación de este estudio para tal se presenta las siguientes ecuaciones, según [17]:

$$
\begin{gathered}
R_{N O}=\left(\begin{array}{c}
N O \\
C O_{2}
\end{array}\right)=\frac{f}{d}, \\
R_{C O}=\left(\begin{array}{c}
C O \\
C O_{2}
\end{array}\right)=\frac{a}{d}, \\
R_{H C}=\left(\begin{array}{c}
H C \\
C O_{2}
\end{array}\right)=\frac{c}{d},
\end{gathered}
$$

dónde: $\mathrm{R}_{c o}$ : Razón de $\mathrm{CO}$ con respecto al $\mathrm{CO}_{2} ; \mathrm{R}_{\mathrm{HC}}$ : Razón de $\mathrm{HC}$ con respecto al $\mathrm{CO}_{2}$; $\mathrm{R}_{N O}$ : Razón de $\mathrm{NO}$ con respecto al $\mathrm{CO}_{2}$.

De las ecuaciones anteriores se obstiene:

$$
\begin{gathered}
a=d * R_{C O}, \\
c=d * R_{H C}, \\
d=\left(\frac{1}{R_{C O}+3 R_{H C}+1}\right) .
\end{gathered}
$$

Al obtener los valores de $a, c$ y $f$ en las ecuaciones anteriores se pretende analizar los factores de emisión (EF') en gramos de contaminante por gramo de combustible consumido según [19]:

$$
\begin{gathered}
E F^{\prime}{ }^{\prime}{ }_{C O}=a * \frac{M W_{C O}}{M W_{\text {Fuel }}}=\frac{R_{C O}}{R_{C O}+3 R_{H C}+1} * \frac{M W_{C O}}{M W_{F u e l}}, \\
E F^{\prime}{ }_{H C}=a * \frac{M W_{H C}}{M W_{\text {Fuel }}}=\frac{R_{H C}}{R_{C O}+3 R_{H C}+1} * \frac{M W_{H C}}{M W_{\text {Fuel }}}, \\
E F^{\prime}{ }^{\prime}{ }^{\prime} O=a * \frac{M W_{N O}}{M W_{\text {Fuel }}}=\frac{R_{N O}}{R_{C O}+3 R_{H C}+1} * \frac{M W_{N O}}{M W_{\text {Fuel }}} .
\end{gathered}
$$

Se considera como base principal del combustible al octano $\mathrm{C} 8 \mathrm{H} 18$ (equivalente $\mathrm{CH} 2.25$ ), se presenta la ecuación del peso molecular del combustible según [17].

$$
M W_{\text {Fuel }}=\frac{12 g C}{\text { mol C }}\left(\frac{1 \mathrm{~mol} \mathrm{C}}{\text { mol Fuel }}\right)+\frac{1 \mathrm{gH}}{\text { mol H }} *\left(\frac{2.25 \mathrm{~mol} \mathrm{H}}{\text { mol Fuel }}\right),
$$




$$
M W_{\text {Fuel }}=14.25 * \frac{g \text { Fuel }}{\text { mol Fuel }}
$$

La densidad del combustible $(\mathrm{kg} / \mathrm{m} 3)$ y consumo por distancia recorrida $\mathrm{FC}(\mathrm{m} 3 / \mathrm{km})$, se obtiene el factor de emisión en gramos de cada contaminante por kilómetro recorrido, como se muestra en las ecuaciones, las cuales se ejecutan para el cálculo de factores de emisión de los vehículos de prueba que son proporcionados por el análisis ON BOARD [17].

$$
\begin{gathered}
E F=E F^{\prime} * \delta_{F u e l} * F C, \\
F C O=\frac{g_{C O}}{K m}=\frac{28 \frac{\% C O}{\% C O_{2}}}{\frac{\% C O}{\% C O_{2}}+\left(3 \frac{\% H C}{\% C O_{2}}\right)+1} * \frac{\delta_{F u e l} * F C}{0.01425}, \\
F H C=\frac{g_{H C}}{K m}=\frac{42 \frac{\% H C}{\% C O_{2}}}{\frac{\% C O}{\% C O_{2}}+\left(3 \frac{\% H C}{\% C O_{2}}\right)+1} * \frac{\delta_{F u e l} * F C}{0.01425} \\
F N O=\frac{g_{N O}}{K m}=\frac{30 \frac{\% N O}{\% C O_{2}}}{\frac{\% C O}{\% C O_{2}}+\left(3 \frac{\% H C}{\% C O_{2}}\right)+1} * \frac{\delta_{F u e l} * F C}{0.01425}
\end{gathered}
$$

La densidad del combustible $(\mathrm{kg} / \mathrm{m} 3)$ y consumo por distancia recorrida $\mathrm{FC}(\mathrm{m} 3 / \mathrm{km})$, se obtiene el factor de emisión en gramos de cada contaminante por kilómetro recorrido, las Ecuaciones 22, 23, 24 y 25 se ejecutan para el cálculo de factores de emisión del vehículo de prueba, que son proporcionados por el análisis On Board [17].

$$
\begin{gathered}
E F=E F^{\prime} * \delta_{F u e l} * F C \\
F C O=\frac{g_{C O}}{K m}=\frac{28 \frac{\% C O}{\% C O_{2}}}{\frac{\% C O}{\% C O_{2}}+\left(3 \frac{\% C C}{\% C O_{2}}\right)+1} * \frac{\delta_{F u e l} * F C}{0.01425} \\
F H C=\frac{g_{H C}}{K m}=\frac{42 \frac{\% H C}{\% C O_{2}}}{\frac{\% C O}{\% C O_{2}}+\left(3 \frac{\% H C}{\% C O_{2}}\right)+1} * \frac{\delta_{F u e l} * F C}{0.01425} \\
F N O x=\frac{30 \frac{\% N O}{\% C O_{2}}}{K m}=\frac{g_{F u e l} * F C}{0.01425}
\end{gathered}
$$

\subsection{Cálculo de la potencia específica del vehículo (VSP)}

EI VSP de la Ecuación 26, traduce cuantitativamente los parámetros característicos de un modo de conducción a niveles de demanda energética del motor como menciona 
[20]. Además, Tolvett [21], corrobora que el VSP posee la ventaja de ser una unidad básica, permitiendo comparaciones entre datos obtenidos en distintos sitios.

$$
V S P=V\left(1.1 \alpha+g \gamma+C_{R}\right)+C_{D} V^{3}\left[\frac{W}{k g}\right],
$$

donde: V: Velocidad del vehículo (m/s); [: Aceleración del vehículo (m/s2); g: Gravedad $(\mathrm{m} / \mathrm{s})$; \: Elevación vertical del vehículo y relación de distancia horizontal; $C R$ : Coeficiente de resistencia a la rodadura; $C D$ : Coeficiente de arrastre dinámico.

En la Tabla 6 , se observa que los modos 1 y 2 corresponden a gamas de potencia negativa, lo que significa que puede corresponder a un frenado o descenso (respectivamente aceleración e inclinación negativas), que en estas circunstancias es posible regenerar energía. En el modo 3 engloba puntos de VSP nulo, asociados en su mayoría a situaciones de "ralentî", con el coche parado (velocidad nula) [22].

\section{Table 6}

Definición de los modos VSP.

\begin{tabular}{l|l|l|l} 
Modo VSP & Gama VSP $[\mathbf{w} / \mathbf{k g}]$ & Modo VSP & Gama VSP $[\mathbf{w} / \mathbf{k g}]$ \\
\hline $\mathbf{1}$ & $\mathrm{VSP}<-2$ & $\mathbf{8}$ & $13 \leq \mathrm{VSP}<16$ \\
\hline $\mathbf{2}$ & $-2 \leq \mathrm{VSP}<0$ & $\mathbf{9}$ & $16 \leq \mathrm{VSP}<19$ \\
\hline $\mathbf{3}$ & $0 \leq \mathrm{VSP}<1$ & $\mathbf{1 0}$ & $19 \leq \mathrm{VSP}<23$ \\
$\mathbf{4}$ & $1 \leq \mathrm{VSP}<4$ & $\mathbf{1 1}$ & $23 \leq \mathrm{VSP}<28$ \\
$\mathbf{5}$ & $4 \leq \mathrm{VSP}<7$ & $\mathbf{1 2}$ & $28 \leq \mathrm{VSP}<33$ \\
$\mathbf{6}$ & $7 \leq \mathrm{VSP}<10$ & $\mathbf{1 3}$ & $33 \leq \mathrm{VSP}<39$ \\
$\mathbf{7}$ & $10 \leq \mathrm{VSP}<13$ & $\mathbf{1 4}$ & $39 \leq \mathrm{VSP}$
\end{tabular}

\subsubsection{VSP ecopaís}

En la Tabla 7, se presentan los datos de potencia específica del vehículo, las velocidades oscilan de 37 hasta $74 \mathrm{~km} / \mathrm{hr}$ y en las aceleraciones de 0,10 hasta

$0,16 \mathrm{~m} / \mathrm{s} 2$, existe un mejor desempeño en la ruta plano ciudad ya que sus valores de VSP tienen una variancia del 2 al 3\%, mientras que en los otros tramos hay gran diferencia entre los datos.

\subsubsection{VSP Extra + Ferox}

En la Tabla 8, se presentan los datos de potencia específica del vehículo, las velocidades oscilan de 36 hasta $86 \mathrm{~km} / \mathrm{hr}$ y en las aceleraciones de 0,12 hasta

$0,16 \mathrm{~m} / \mathrm{s} 2$, en el tramo de ascenso carretera hay una variancia del 2 al $11 \%$, debido a que existe una mayor velocidad y aceleración. 
Table 7

Potencia específica del vehículo con el combustible Ecopaís.

\begin{tabular}{|c|c|c|c|c|c|c|c|}
\hline \multirow[b]{2}{*}{ TRAMOS } & \multicolumn{7}{|c|}{ PRUEBAS VSP (w/kg) } \\
\hline & 1 & 2 & 3 & 4 & 5 & 6 & 7 \\
\hline Ascenso Carretera & 47,08 & 45,22 & 43,15 & 44,31 & 46,39 & 38,88 & 43,84 \\
\hline Plano Ciudad & 22,51 & 21,18 & 21,90 & 21,11 & 19,93 & 21,18 & 21,70 \\
\hline Ascenso Ciudad & 28,81 & 27,39 & 25,28 & 24,16 & 30,41 & 23,45 & 26,73 \\
\hline
\end{tabular}

Table 8

Potencia específica del vehículo con el combustible Extra + Ferox.

\begin{tabular}{|c|c|c|c|c|c|c|c|}
\hline \multirow[b]{2}{*}{ TRAMOS } & \multicolumn{7}{|c|}{ PRUEBAS VSP (w/kg) } \\
\hline & 1 & 2 & 3 & 4 & 5 & 6 & 7 \\
\hline Ascenso Carretera & 55,65 & 46,25 & 44,76 & 49,31 & 45,50 & 50,86 & 52,44 \\
\hline Plano Ciudad & 23,38 & 21,18 & 19,17 & 25,68 & 23,31 & 24,29 & 22,93 \\
\hline Ascenso Ciudad & 29,35 & 25,44 & 27,39 & 22,89 & 28,04 & 22,26 & 25,44 \\
\hline
\end{tabular}

\subsubsection{VSP Ecopaís + Ferox}

En la Tabla 9, se presentan los datos de potencia específica del vehículo, las velocidades oscilan de 39 hasta $72 \mathrm{~km} / \mathrm{hr}$ y en las aceleraciones de 0,12 hasta $0,16 \mathrm{~m} / \mathrm{s}^{2}$, existe un mejor desempeño en la ruta plano ciudad ya que sus valores de VSP tienen una variancia del 1 al 3\%, mientras que en los otros tramos hay gran diferencia entre los datos.

Table 9

Potencia específica del vehículo con el combustible Ecopaís + Ferox.

\begin{tabular}{|c|c|c|c|c|c|c|c|}
\hline \multirow[b]{2}{*}{ TRAMOS } & \multicolumn{7}{|c|}{ PRUEBAS VSP (w/kg) } \\
\hline & 1 & 2 & 3 & 4 & 5 & 6 & 7 \\
\hline Ascenso Carretera & 55,65 & 52,44 & 44,76 & 45,50 & 46,25 & 43,28 & 47,77 \\
\hline Plano Ciudad & 21,61 & 19,90 & 20,75 & 19,90 & 22,48 & 23,38 & 21,18 \\
\hline Ascenso Ciudad & 29,35 & 25,44 & 22,89 & 27,39 & 24,16 & 25,44 & 27,39 \\
\hline
\end{tabular}

2.8. Datos de torque y potencia

2.8.1. Combustible Extra + Ferox - torque y potencia 


\section{Prueba 1}

Con el combustible Extra + Ferox el torque en la prueba 1 fue de 104,8 Nm a 5305 RPM y su potencia fue de $87,5 \mathrm{Hp}$ a 6070 RPM, mostrada en la Figura 20.

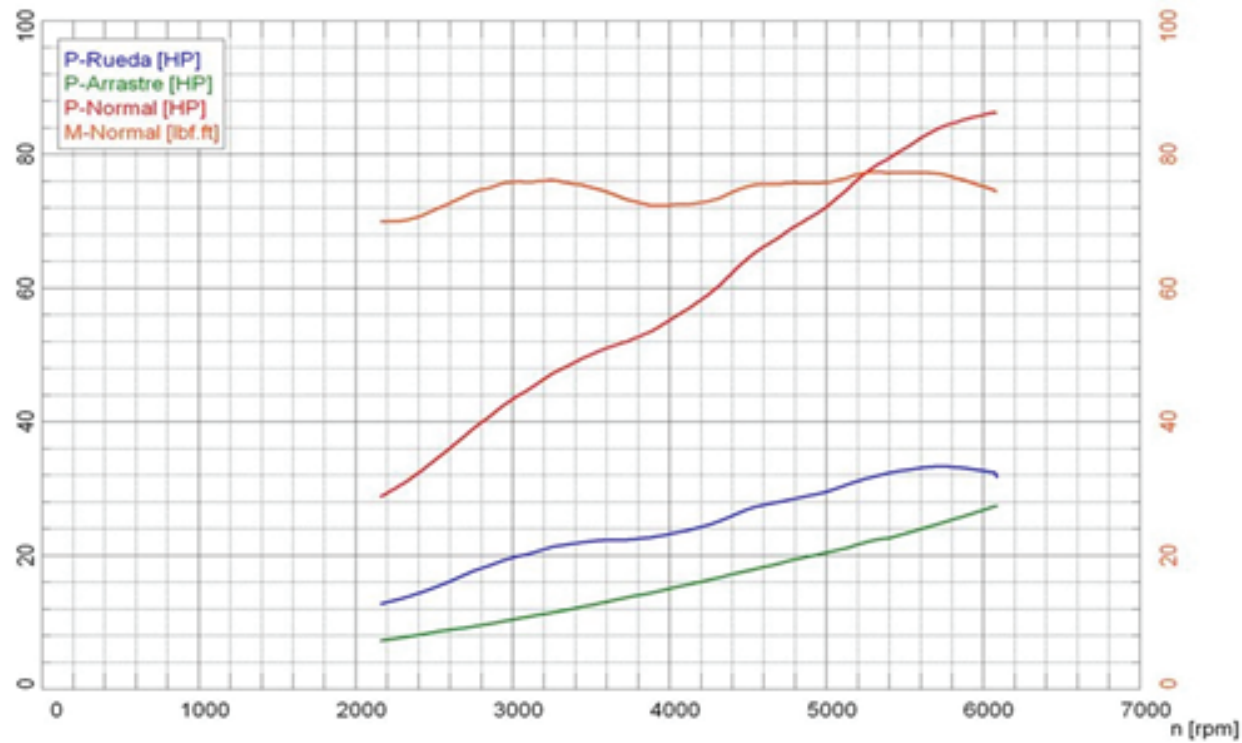

Figure 20

Potencia con el combustible Extra Ferox-Prueba 1.

\section{Prueba 2}

Con el combustible Extra + Ferox el torque en la prueba 2 fue de 107,25 Nm a 5380 RPM y su potencia fue de 87,3 $\mathrm{Hp}$ a 6080 RPM, mostrada en la Figura 21.

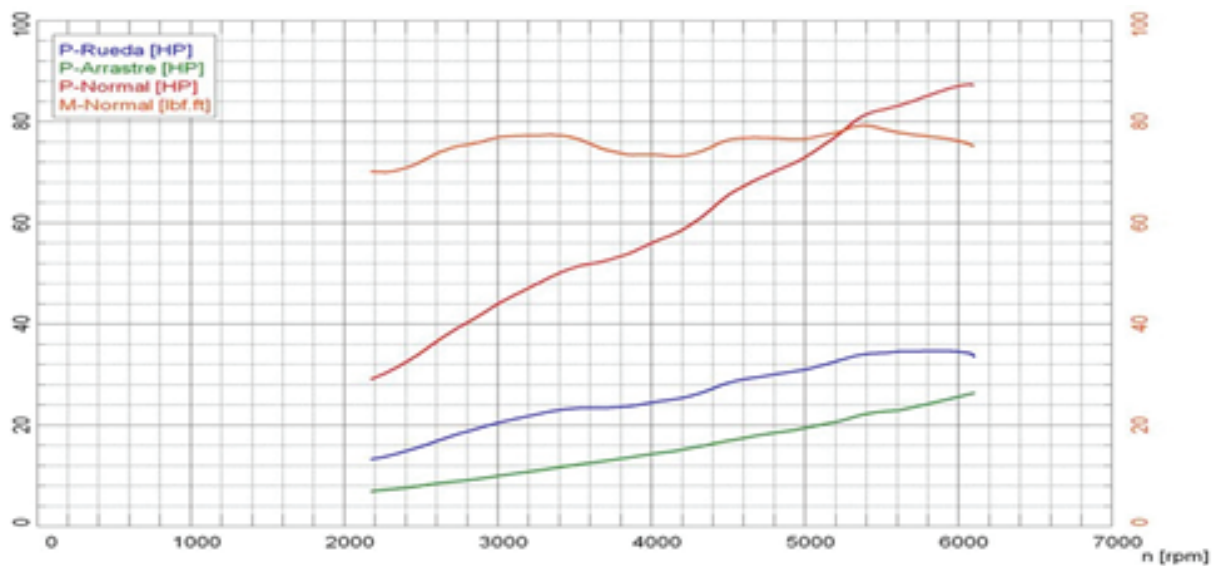

Figure 21

Potencia con el combustible Extra Ferox-Prueba 2 


\section{Prueba 3}

Con el combustible Extra + Ferox el torque en la prueba 3 fue de 107,52 Nm a 5455 RPM y su potencia fue de 87,5 Hp a 6085 RPM, mostrada en la Figura 22.

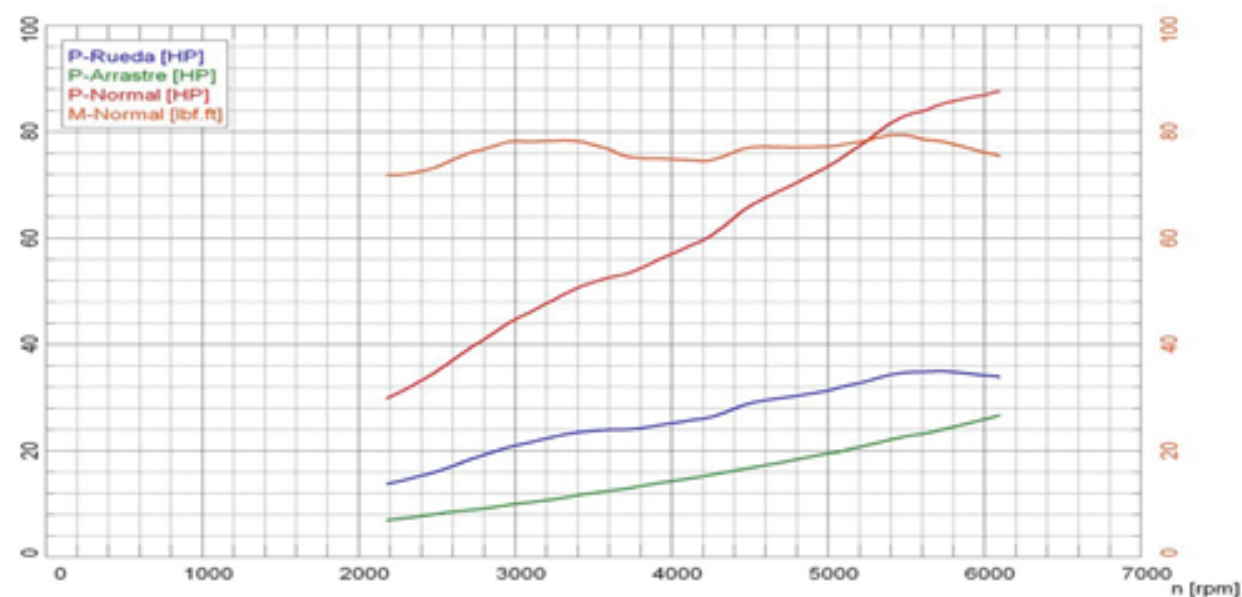

Figure 22

Potencia con el combustible Extra Ferox-Prueba 3.

\subsubsection{Combustible Ecopaís - torque y potencia}

\section{Prueba 1}

Con el combustible Ecopaís el torque en la prueba 1 fue de 108,97 Nm a 5405 RPM y su potencia fue de $88,3 \mathrm{Hp}$ a 6065 RPM, mostrada en la Figura 23.

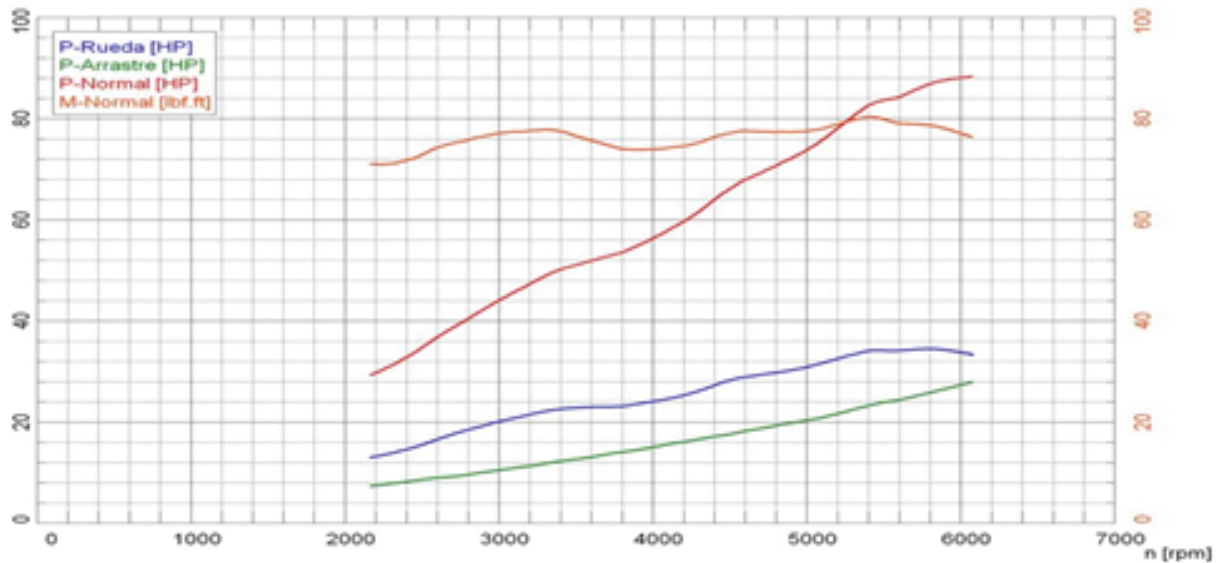

Figure 23

Potencia con el combustible Ecopaís-Prueba 1. 


\section{Prueba 2}

Con el combustible Extra Ferox el torque en la prueba 2 fue de 108,97 Nm a 5365 RPM y su potencia fue de 88,2 Hp a 6065 RPM, mostrada en la Figura 24.

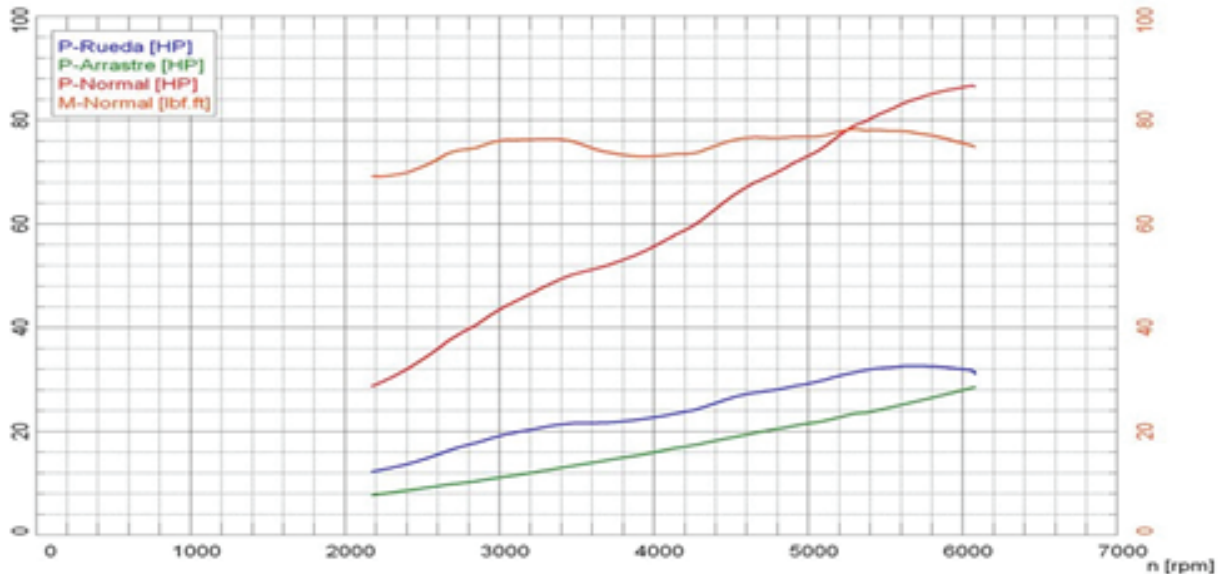

Figure 24

Potencia con el combustible Ecopaís-Prueba 2.

\section{Prueba 3}

En este caso con el combustible Ecopaís el torque en la prueba 3 fue de $109 \mathrm{Nm}$ a 5435 RPM y su potencia fue de 89,3 Hp a 6070 RPM, mostrada en la Figura 25.

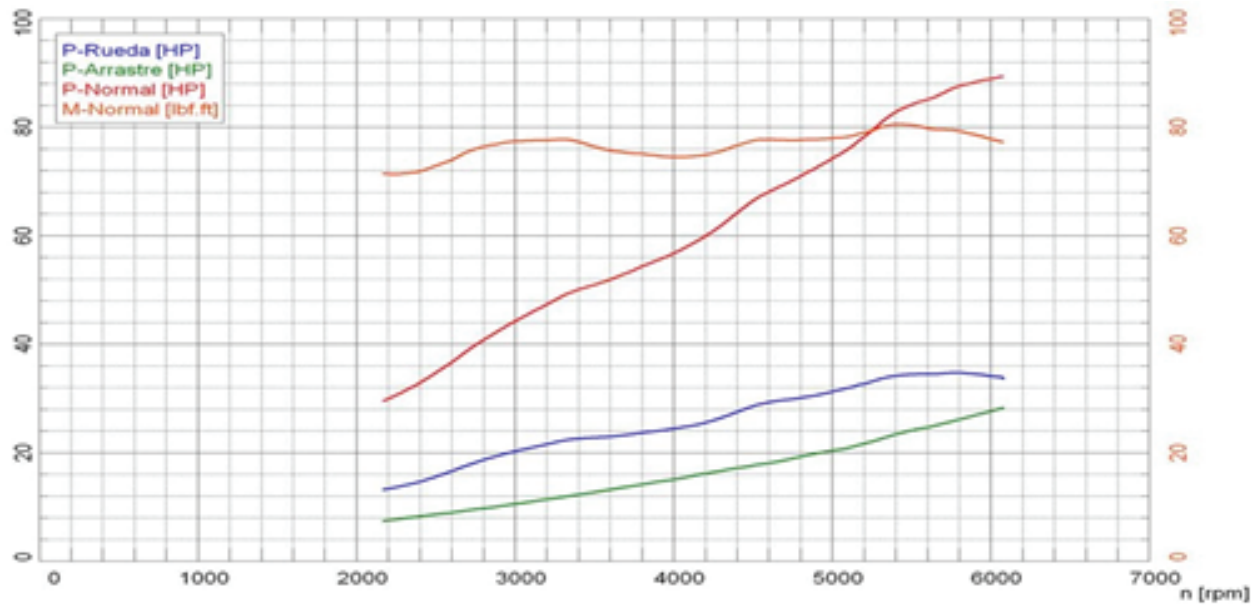

Figure 25

Potencia con el combustible Ecopaís-Prueba 3.

2.8.3. Combustible Ecopaís + Ferox - torque y potencia 


\section{Prueba 1}

Con el combustible Ecopaís + Ferox el torque en la prueba 1 fue de 107,79 Nm a 5485 RPM y su potencia fue de 89,3 Hp a 6095 RPM, mostrada en la Figura 26.

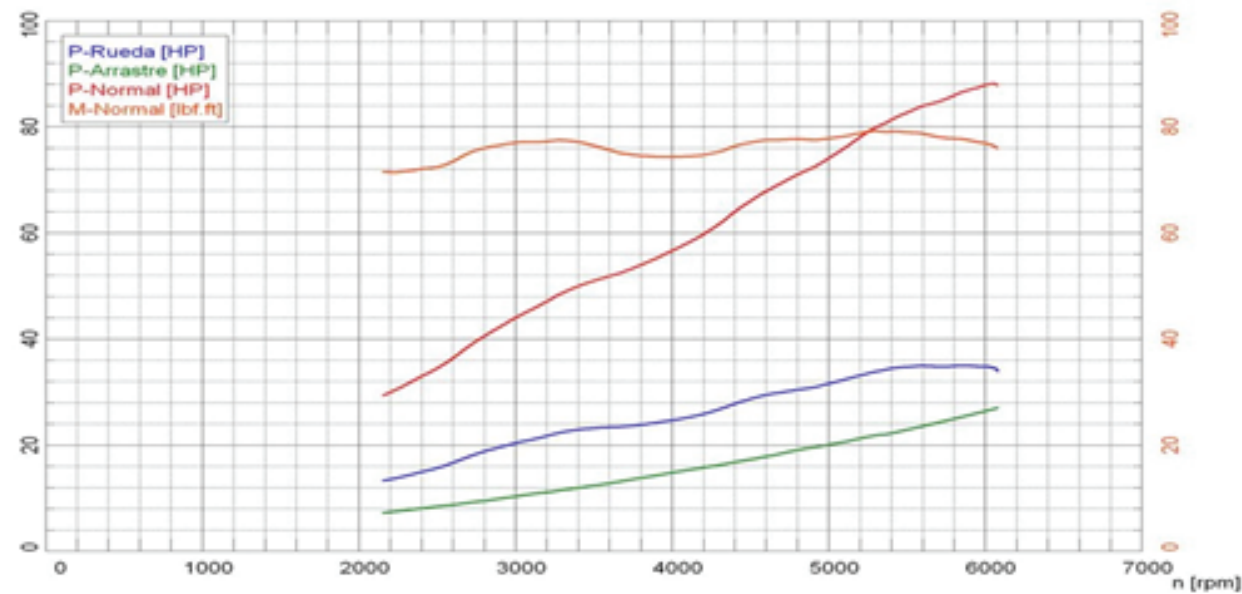

Figure 26

Potencia con el combustible Ecopaís Ferox-Prueba 1.

\section{Prueba 2}

Con el combustible Ecopaís + Ferox el torque en la prueba 2 fue de 108,47 Nm a 5340 RPM y su potencia fue de 87,9 Hp a 6070 RPM, mostrada en la Figura 27.

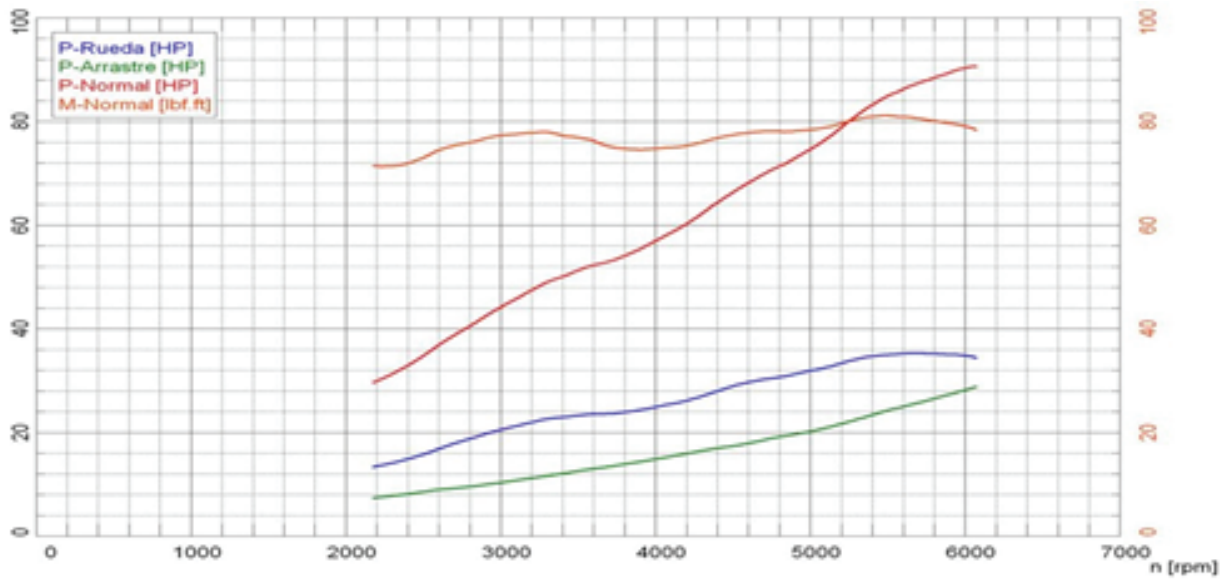

Figure 27

Potencia con el combustible Ecopaís Ferox-Prueba 2. 


\section{Prueba 3}

Con el combustible Ecopaís + Ferox el torque en la prueba 3 fue de 107,25 Nm a 5285 RPM y su potencia fue de $88 \mathrm{Hp}$ a 6050 RPM, mostrada en la Figura 28.

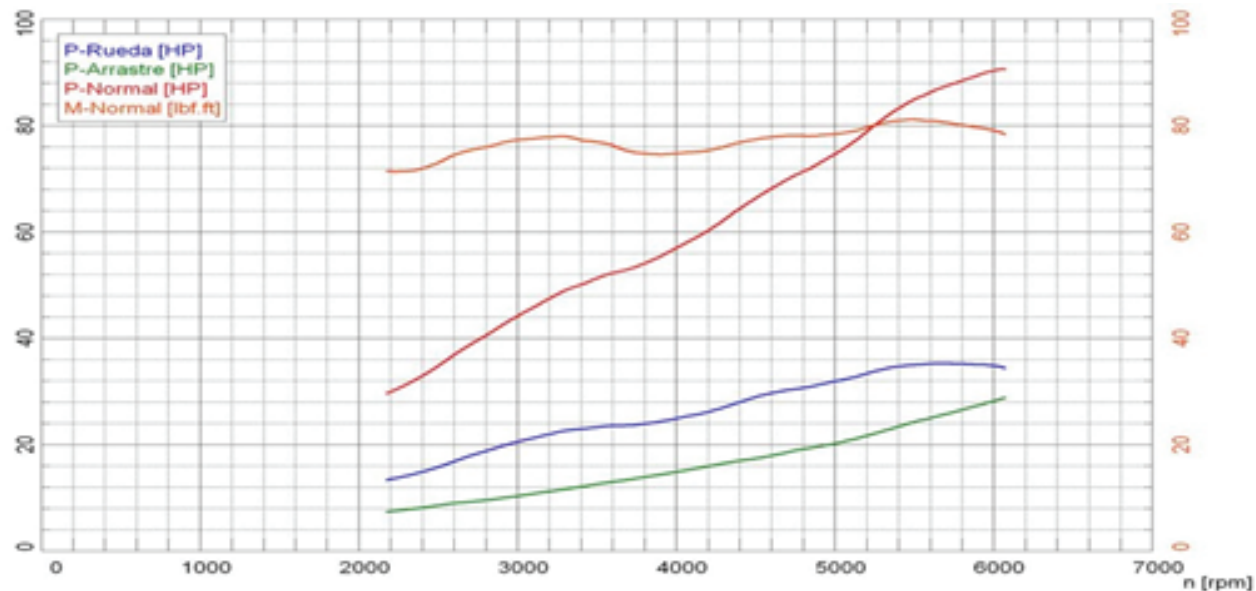

Figure 28

Potencia con el combustible Ecopaís Ferox-Prueba 3.

\section{Resultados y Discusión}

\subsection{Factores de emisiones}

Con el estudio de [23], quienes exponen investigaciones con combustibles E5 donde realizan pruebas dinámicas de gases; en el caso del CO que hay una reducción del $100 \%$ con el uso de combustible E5, en los HC existe una disminución del 65\% con el uso del combustible E5 comparado con la gasolina común, en los $\mathrm{CO} 2$ existe una reducción de 1\% con el combustible E5, a 2880 m.s.n.m. En las Tablas 10, 11 y 12 se muestran los factores de emisión de los diferentes combustibles en la ruta establecida, del vehículo de pruebas.

Table 10

Factores de contaminación del combustible Ecopaís.

$\mathrm{FCO}(\mathrm{g} / \mathrm{km})$

$\mathrm{FHC}(\mathrm{g} / \mathrm{km})$

FNOX (g/km)
7,161

0,147

0,148 


\section{Table 11}

Factores de contaminación del combustible Extra + Ferox.

$\mathrm{FCO}(\mathrm{g} / \mathrm{km})$
$\operatorname{FHC}(\mathrm{g} / \mathrm{km})$
$\operatorname{FNOX}(\mathrm{g} / \mathrm{km})$

9,458
0,131
0,143

Table 12

Factores de contaminación del combustible Ecopaís + Ferox.

FCO $(\mathrm{g} / \mathrm{km})$
$\mathrm{FHC}(\mathrm{g} / \mathrm{km})$
$\mathrm{FNOX}(\mathrm{g} / \mathrm{km})$

7,825
0,146
0,144

\subsubsection{Factores de emisiones de $\mathrm{CO}$}

En la Figura 29, muestra que el combustible que presenta una diferencia significativa de menos factores de emisión en CO es el Ecopaís de 1\% y 2,5\% en comparación Ecopaís + Ferox y Extra + Ferox respectivamente. De igual forma mencionan Kannan et al. [24], que entre mayor sea el porcentaje de etanol, menor es la cantidad de CO que emite al ambiente, con la E5 hay una reducción significativa del monóxido de carbono.

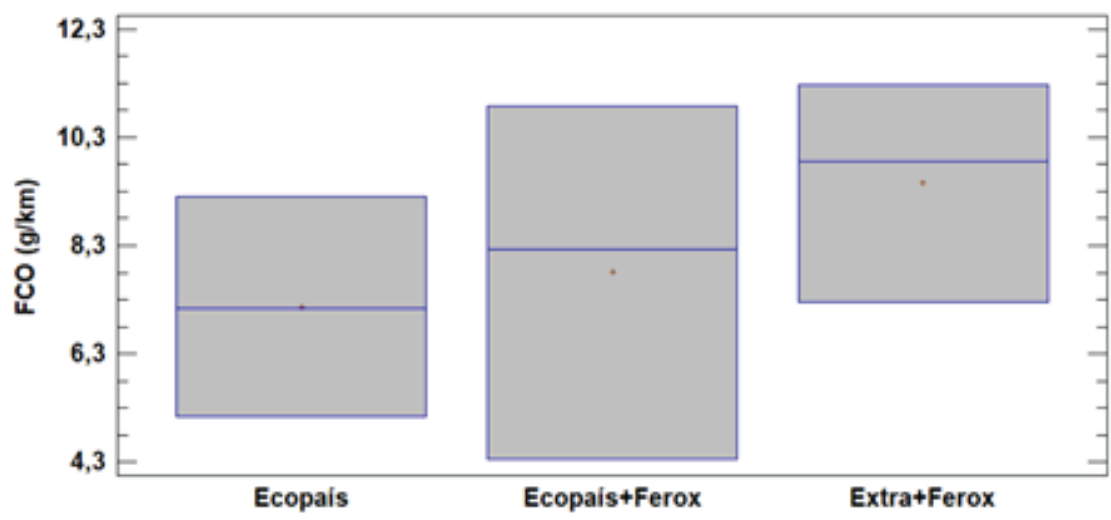

Figure 29

Factores de emisión de CO con 3 combustibles.

\subsubsection{Factores de Emisiones de HC}

En la Figura 30, el mejor resultado con menores emisiones en los combustibles que contienen aditivos y etanol, en el caso de Ecopaís + Ferox y en Extra + Ferox con un 0\% y $1 \%$ respectivamente, en comparación del combustible Ecopaís. El estudio expuesto 
por Dogan et al. [25], contribuye que los valores en la cantidad de HC se reduce a medida que el porcentaje de etanol sea menor a E30 (30\% de etanol).

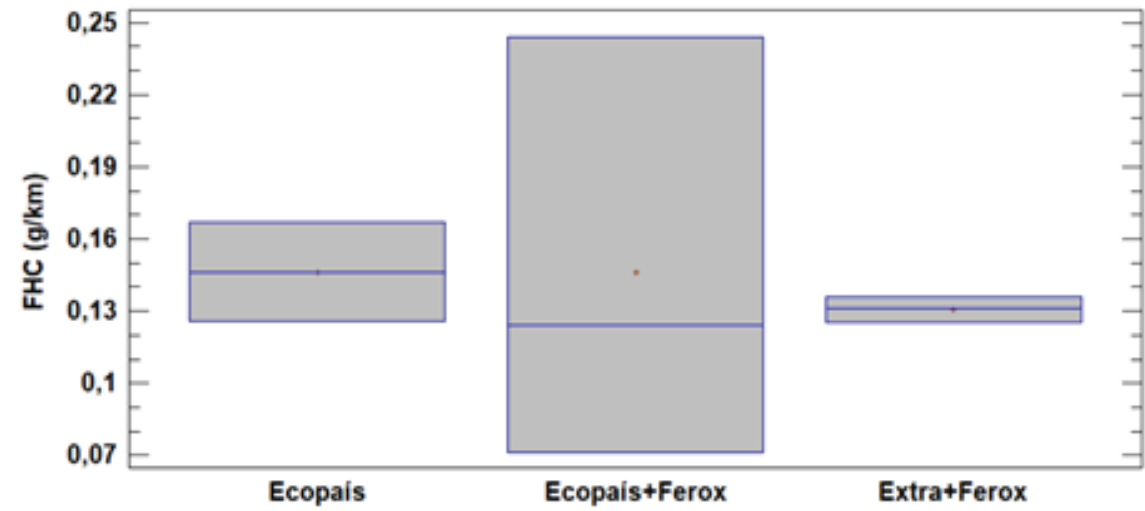

Figure 30

Factores de emisión de HC con 3 combustibles.

\subsubsection{Factores de Emisiones de NOx}

En la Figura 31, se aprecia los factores de emisión para el NOx, donde los resultados significativos son favorables para los combustibles con aditivos en el caso de Extra + Ferox un 6\% y en Ecopaís + Ferox un 4\% en comparación del combustible Ecopaís. El estudio expuesto por Dogan et al. [25], mencionan que el valor más alto para la emisión de NOx se mide para el combustible que tenga $0 \%$ de etanol, mientras que los combustibles con mezcla etanol tienen bajos valores de emisión de NOx.

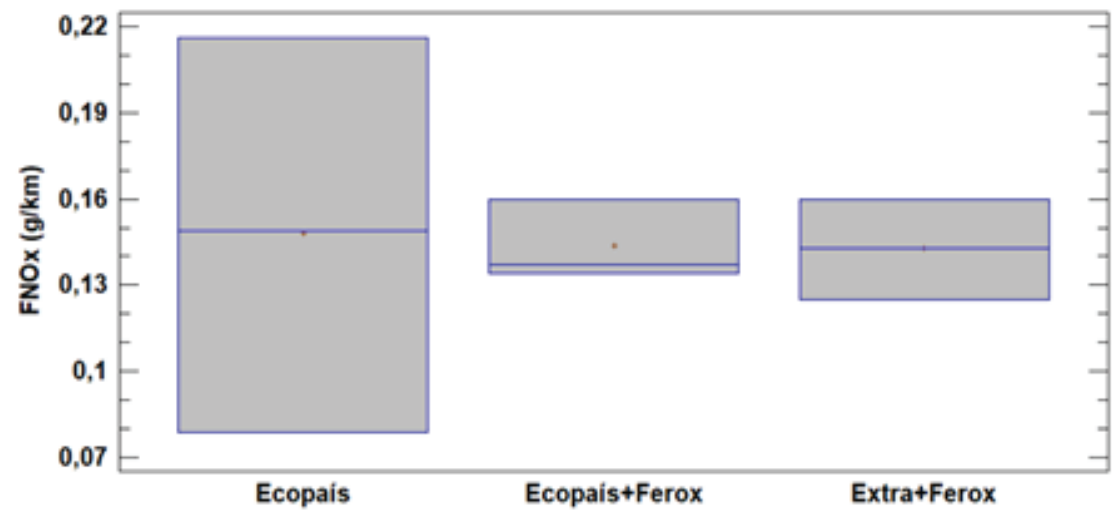

Figure 31

Factores de emisión de NOx con 3 combustibles. 


\subsection{Consumo de combustible}

El rendimiento de combustible para el vehículo en la ruta estimada se encuentra en el rango de $7,80 \mathrm{~km} / \mathrm{L}$ hasta $9,14 \mathrm{~km} / \mathrm{L}$ observado en la Figura 32 . Se define que hay 0,5\% hasta 1,3\% de ahorro de combustible con el combustible Ecopaís + Ferox y Extra + Ferox a diferencia de los otros combustibles. El factor tráfico, horas pico y cambios climáticos se relacionan mucho con el consumo de combustible.

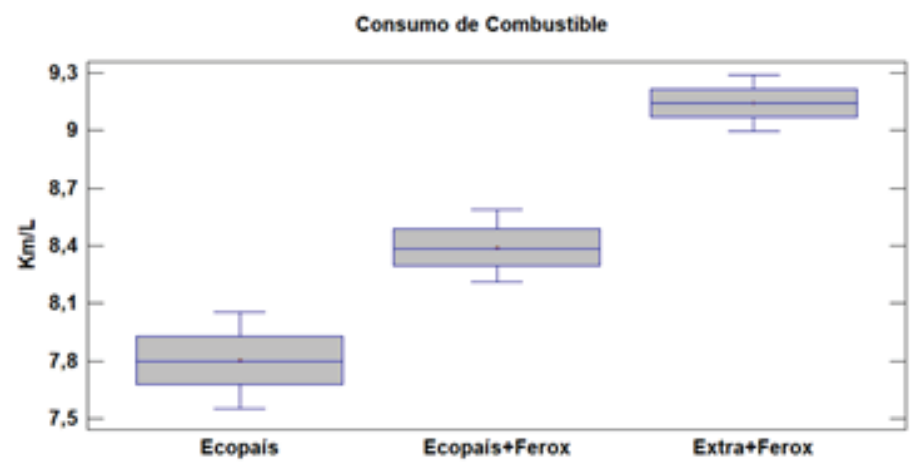

Figure 32

Consumo de combustible con 3 tipos.

\subsection{Torque o Par motor}

En la Figura 33 se observa el comportamiento del Torque usando los combustibles anteriormente mencionados, donde se sustenta un valor de $109 \mathrm{Nm}$ a $5600 \mathrm{Rpm}$, en el carburante Ecopaís. Teniendo un aumento significativo del $2 \%$ en su Par motor con respecto al combustible Extra + Ferox y con respecto al Ecopaís + Ferox el 1,1\%. Con el aditivo Ferox, las dos mezclas de gasolinas no tienen algún beneficio significativo en el Torque del motor. Lo expuesto por Rocha y Zambrano [26], al utilizar aditivo solido en la mezcla hay una reducción del $2 \%$ en el par motor.

\subsection{Potencia}

Se verifica en la Figura 34, un incremento gradual del 1,6\% de 88,6 Hp a $6000 \mathrm{Rpm}$ utilizando el combustible Ecopaís con respecto al Extra + Ferox. También muestra que la potencia máxima alcanzada por dicho combustible se da a menores regímenes de giro. Lo que menciona [27], corrobora que el torque y la potencia indicados no experimentan un cambio resaltable en mezclas con altos contenidos de etanol. 


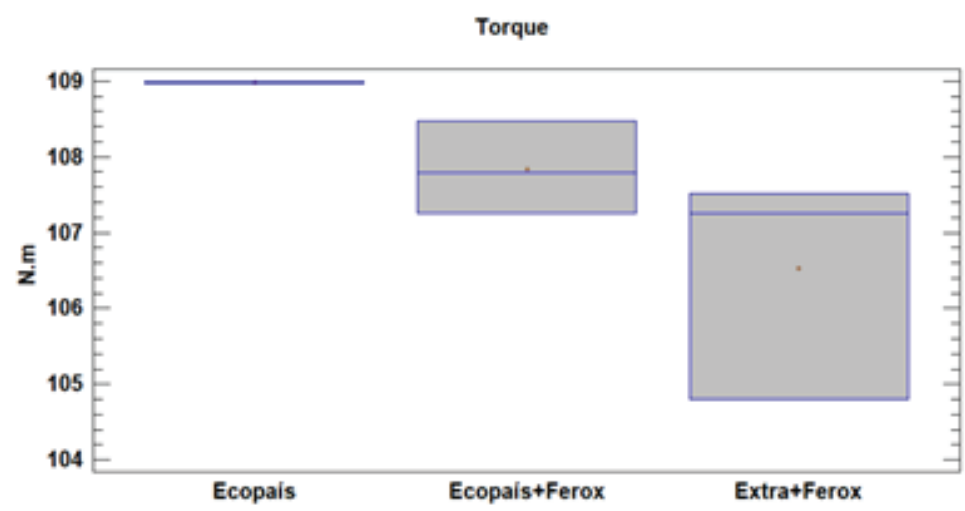

Figure 33

Comparación del Torque con 3 tipos de combustibles.

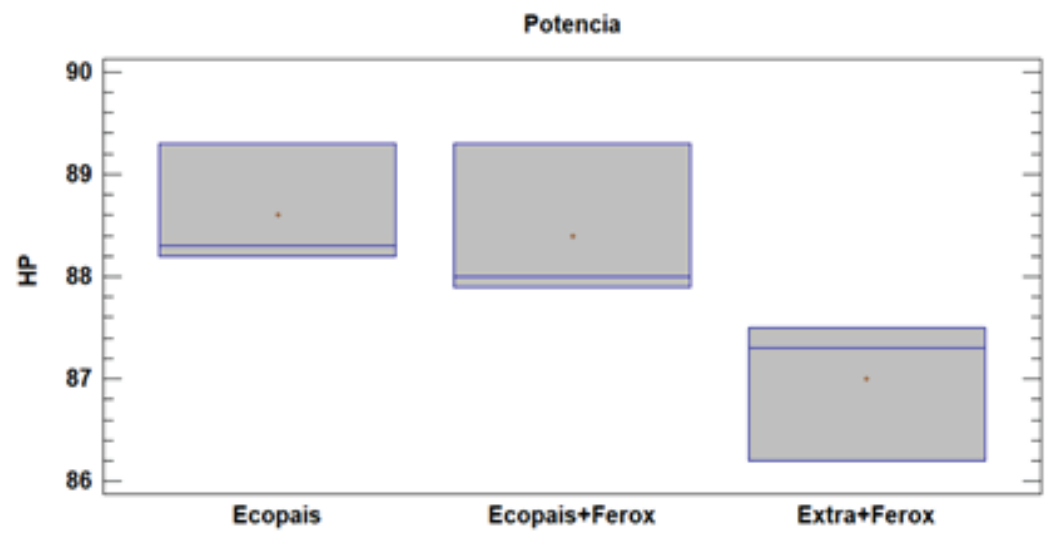

Figure 34

Comparación de Potencia con 3 tipos de combustibles.

\subsection{VSP}

En este tipo de prueba se toma tres tramos cada una con siete muestras, para tres tipos de combustibles. Los valores considerados para el coeficiente de arrastre dinámico (CD) y coeficiente de resistencia a la rodadura (CR) son $0.132\left(\mathrm{~m} / \mathrm{s}^{2}\right)$ y $0.000302\left(\mathrm{~m}^{-1}\right)$ respectivamente, valores expuestos por [22]. Y en el caso de la velocidad, aceleración y pendiente se toma valores medios para el cálculo del VSP, mostrados en la Tablas 13,14 y 15.

\subsubsection{Potencia específica del vehículo para carretera en ascenso}

En este tramo de ruta mostrado en la Figura 35, las circunstancias presentadas de la forma de conducción, aceleración y velocidad son favorables para dos combustibles Ecopaís, Ecopaís + Ferox, donde existe una diferencia significativa con el combustible Extra + Ferox del 5 al 6\%, razón por la cual en este tramo existe una gran influencia 


\section{Table 13}

VSP del combustible Ecopaís.

\begin{tabular}{|c|c|c|c|c|c|c|c|}
\hline \multirow[b]{2}{*}{ Tramos } & \multicolumn{7}{|c|}{ PRUEBAS VSP (w/kg) } \\
\hline & 1 & 2 & 3 & 4 & 5 & 6 & 7 \\
\hline $\begin{array}{l}\text { Ascenso } \\
\text { carretera }\end{array}$ & 47,08 & 45,22 & 43,15 & 44,31 & 46,39 & 38,88 & 43,84 \\
\hline $\begin{array}{l}\text { Plano } \\
\text { ciudad }\end{array}$ & 22,51 & 21,18 & 21,90 & 21,11 & 19,93 & 21,18 & 21,70 \\
\hline $\begin{array}{l}\text { Ascenso } \\
\text { ciudad }\end{array}$ & 28,81 & 27,39 & 25,28 & 24,16 & 30,41 & 23,45 & 26,73 \\
\hline
\end{tabular}

Table 14

VSP del combustible Extra + Ferox.

\begin{tabular}{|c|c|c|c|c|c|c|c|}
\hline \multirow[t]{2}{*}{ Tramos } & 1 & 2 & 3 & 4 & 5 & 6 & 7 \\
\hline & \multicolumn{7}{|c|}{ PRUEBAS VSP (w/kg) } \\
\hline $\begin{array}{l}\text { Ascenso } \\
\text { carretera }\end{array}$ & 55,65 & 46,25 & 44,76 & 49,31 & 45,50 & 50,86 & 52,44 \\
\hline $\begin{array}{l}\text { Plano } \\
\text { ciudad }\end{array}$ & 23,38 & 21,18 & 19,17 & 25,68 & 23,31 & 24,29 & 22,93 \\
\hline $\begin{array}{l}\text { Ascenso } \\
\text { ciudad }\end{array}$ & 29,35 & 25,44 & 27,39 & 22,89 & 28,04 & 22,26 & 25,44 \\
\hline
\end{tabular}

Table 15

VSP del combustible Ecopaís + Ferox.

\begin{tabular}{|c|c|c|c|c|c|c|c|}
\hline \multirow[b]{2}{*}{ Tramos } & \multicolumn{7}{|c|}{ PRUEBAS VSP (w/kg) } \\
\hline & 1 & 2 & 3 & 4 & 5 & 6 & 7 \\
\hline $\begin{array}{l}\text { Ascenso } \\
\text { carretera }\end{array}$ & 55,65 & 52,44 & 44,76 & 45,50 & 46,25 & 43,28 & 47,77 \\
\hline $\begin{array}{l}\text { Plano } \\
\text { ciudad }\end{array}$ & 21,61 & 19,90 & 20,75 & 19,90 & 22,48 & 23,38 & 21,18 \\
\hline $\begin{array}{l}\text { Ascenso } \\
\text { ciudad }\end{array}$ & 29,35 & 25,44 & 22,89 & 27,39 & 24,16 & 25,44 & 27,39 \\
\hline
\end{tabular}

de tráfico en horas pico. Concordando con [28], la potencia específica del vehículo depende del tramo a estudiar, elementos que varían en determinadas horas del día y altera los resultados de este.

\subsubsection{Potencia específica del vehículo para plano-ciudad}

En la Figura 36 se evidencia una diferencia significativa con el combustible Extra + Ferox del 2\%, mientras con Ecopaís y Ecopaís + Ferox no existe comparación alguna, Corroborando con [28], menciona que la desaceleración genera VSP negativos, pero 


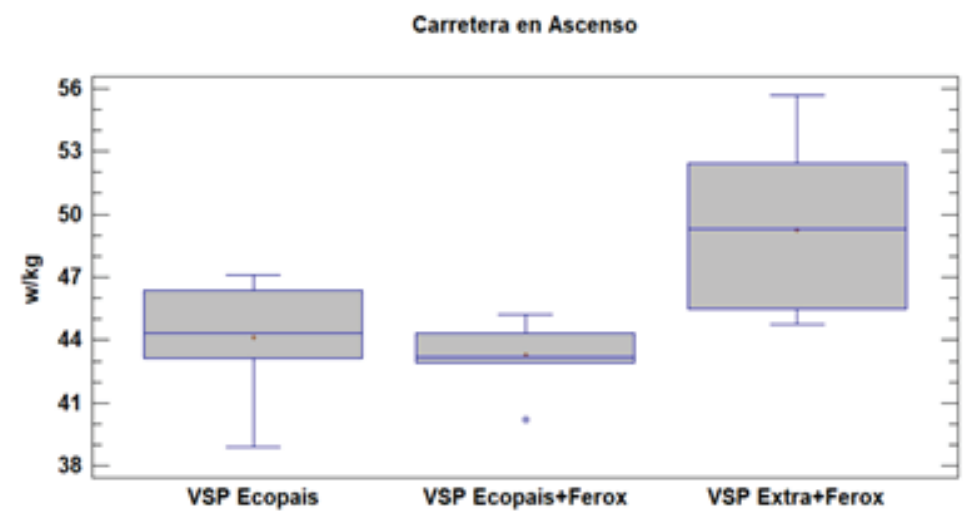

Figure 35

Análisis de Potencia con diferentes combustibles en carretera.

es el hecho de obtener tramos variados que salen de las condiciones normales de operación.

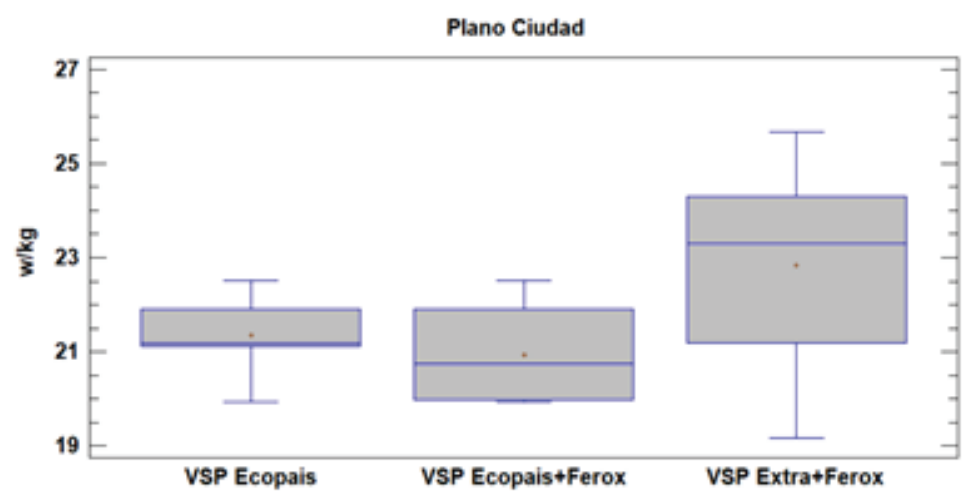

Figure 36

Análisis de potencia con diferentes combustibles en ciudad.

\subsubsection{Potencia específica del vehículo para ciudad-ascenso}

Se observa en la Figura 37, que la desaceleración es un factor importante por el hecho de encontrarse circunstancias de tráfico y señales de tránsito, donde los valores del VSP no tienen ninguna diferencia significa entre los combustibles. Concordando con [29], la implicación de un alto VSP tiene un impacto más sustancial sobre las emisiones de CO que para los otros contaminantes. Esto parece plausible, ya que es probable que un alto VSP, tienda a tener más efecto sobre las emisiones de $\mathrm{CO}$ que por las emisiones de NOx. 


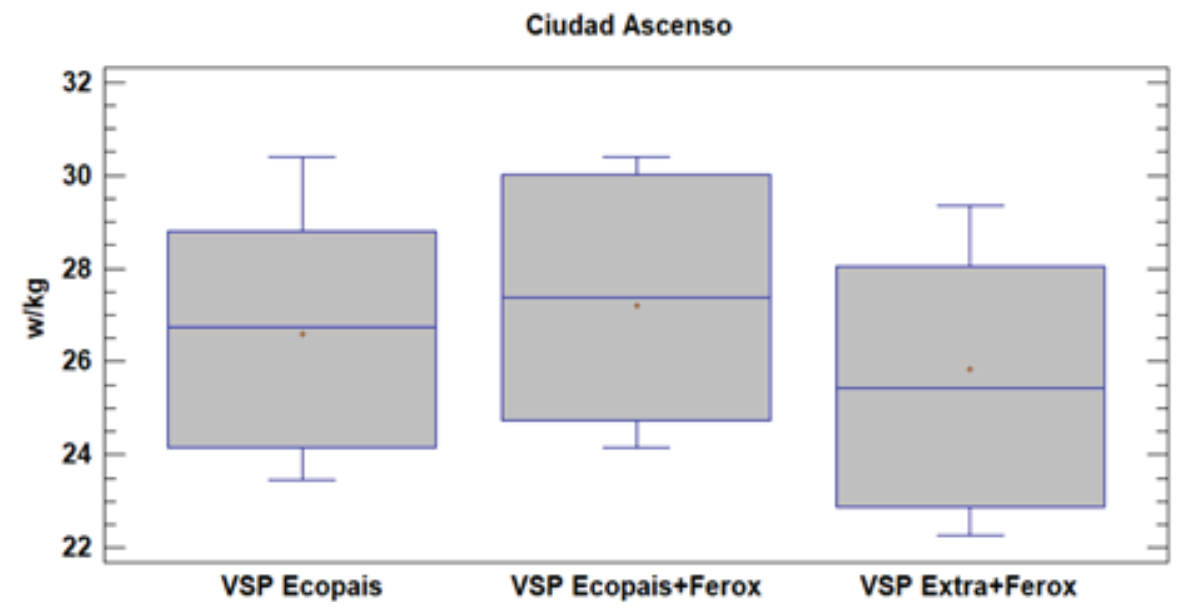

Figure 37

Análisis de potencia con diferentes combustibles en ciudad-ascenso.

\subsection{Análisis Térmico de la bujía de encendido con diferentes com- bustibles}

\subsubsection{Temperatura}

La temperatura promedio del combustible Extra + Ferox es de $527,24^{\circ} \mathrm{C}$, mientras que en Ecopaís es de $599,02^{\circ} \mathrm{C}$, datos obtenidos mediante ANSYS. Y en el caso de la Ecopaís + Ferox su temperatura promedio es de $603,52^{\circ} \mathrm{C}$, dando una diferencia significativa del 4,5\% con el combustible Ecopaís, teniendo la mayor temperatura Ecopaís + Ferox. De acuerdo con [6], la temperatura que se alcanza en la cámara de combustión varía de unos motores a otros en función de la refrigeración, de la relación de compresión, etc. Sin embargo, la temperatura de funcionamiento de una bujía debe mantenerse por encima del límite de autolimpieza $\left({ }^{\circ} \mathrm{C}\right)$ y por debajo del límite de inflamaciones prematuras $\left(900^{\circ} \mathrm{C}\right)$, independientemente del motor que se trate. En la Figura 38 se observa el mayor grado térmico en la bujía.

\subsubsection{Flujo de calor}

En este caso al usar combustible Extra + Ferox es inferior al flujo de calor del combustible Ecopaís, el flujo de calor promedio de estos combustibles es de $48361 \mathrm{~W} / \mathrm{m}^{2}$ y 50534 $\mathrm{W} / \mathrm{m}^{2}$ respectivamente, mientras que en Ecopaís es de $59228 \mathrm{~W} / \mathrm{m}^{2}$, y en el caso de la Ecopaís + Ferox el flujo de calor promedio es de $59.767 \mathrm{~W} / \mathrm{m}^{2}$, existiendo una diferencia del $5 \%$ con el combustible Ecopaís, teniendo el mayor flujo de calor de Ecopaís + Ferox. Según [24], indican que un cuerpo con gran velocidad molecular tiene más temperatura o más nivel de calor que otro. El Estudio confirma que con el uso de dos propuestas de combustible en la que se fijan parámetros comparativos que permiten determinar 


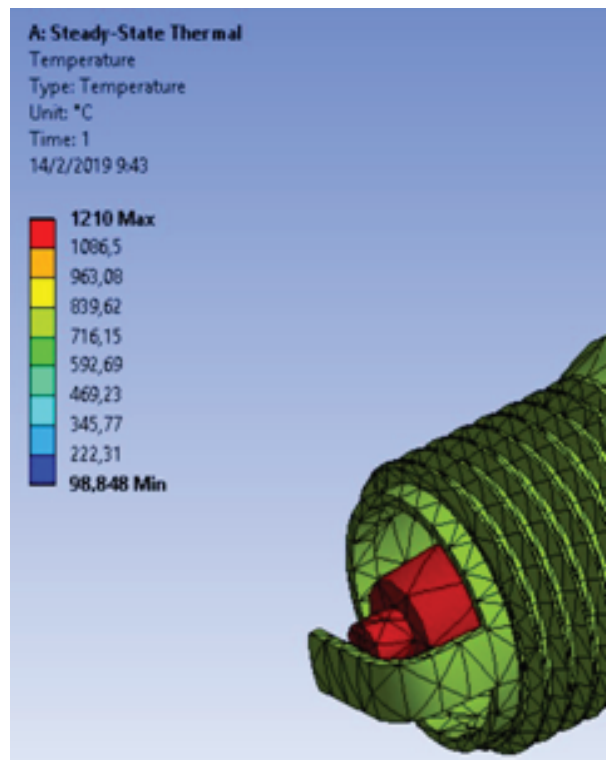

Figure 38

Análisis térmico de la bujía de encendido combustible Ecopaís + Ferox.

dicho nivel de calor, al que se denomina temperatura. En la Figura 39 se observa el mayor flujo de calor en la bujía.

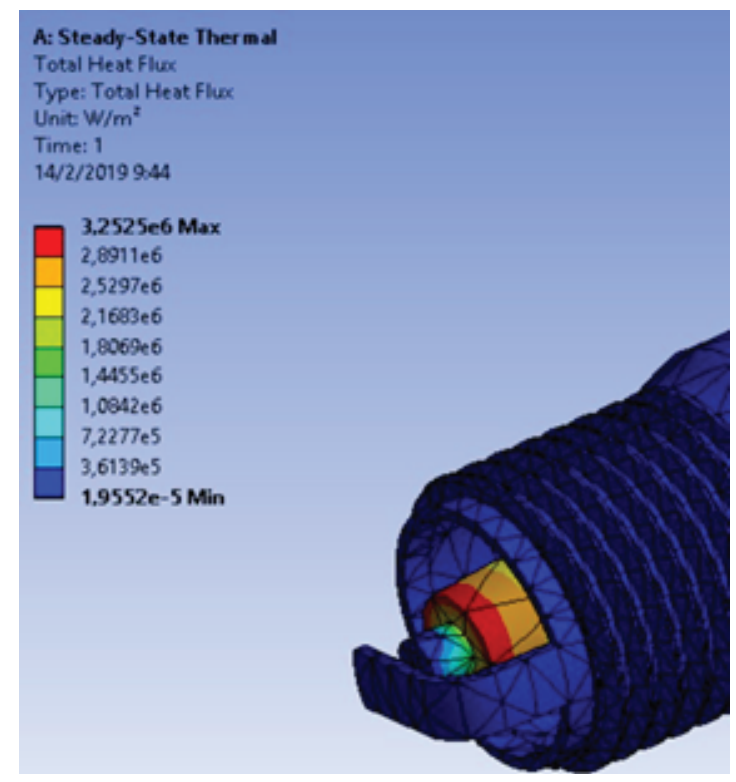

Figure 39

Análisis del flujo de calor del combustible Ecopaís + Ferox. 


\section{Conclusiones}

Se demuestra con este estudio una significativa reducción de emisiones contaminantes del 1 y $2,5 \%$ del FCO con el uso del Ecopaís en comparación del combustible Extra + Ferox y Ecopaís + Ferox respectivamente, en el caso de FHC los combustibles Ecopaís y Ecopaís+Ferox con un 1\% menor en emisiones en comparación al combustible Extra + Ferox, y por último en el FNOx los combustibles que tienen aditivo sólido Extra + Ferox y Ecopaís + Ferox son menores en un 6 y $4 \%$ con respecto al combustible Extra respectivamente.

El software de diseño (NX) y simulación (ANSYS), fue el instrumento que permitió la interacción del componente con 14.977 nodos y 7523 en elementos a estudiar con el mallado automático, consiguiendo así que con los combustibles Ecopaís y Ecopaís + Ferox el mayor flujo de calor con un contraste significativo del $5 \%$, de forma que se nota la máxima temperatura de trabajo de la bujía de encendido y el mayor aprovechamiento de energía térmica expuesto por estos combustibles.

El rendimiento óptimo en consumo de combustible fue el combustible Ecopaís con un valor de 7,80 (km/L), teniendo una diferencia significativa con el combustible Extra + Ferox del 1,3\%, esto representa un ahorro económico. Se denota que los ciclos de conducción y el tipo de ruta también influyen en el consumo.

Con la sistemática de Potencia específica del vehículo (VSP), en el primer y segundo tramo de la ruta los combustibles Ecopaís + Ferox, Ecopaís tienen valores parecidos; mientras que con el combustible Extra + Ferox estos dos combustibles tienen una diferencia del 6 y $4 \%$ respectivamente y en el tramo final ciudad-ascenso no existe diferencia en sus VSP con los combustibles de estudio. Estos datos se pueden obtener en cualquier otro ciclo de manejo, usando la distribución de tiempo VSP correspondiente, para estimar el uso de combustible y el resultado de emisión en un vehículo determinado o una flota de vehículos.

Se sustenta un incremento gradual del 1,6 \% de $88 \mathrm{Hp}$ a $6000 \mathrm{Rpm}$ utilizando el combustible Ecopaís con respecto al Extra + Ferox. También se observa que la potencia máxima alcanzada por dicho combustible se da a menores regímenes de giro. Y comparando con los combustibles Ecopaís y Ecopaís + Ferox, no existe diferencia significativa.

El comportamiento del Par motor o Torque usando los combustibles de estudio, se sustenta un valor de 108,91 Nm a 5402 Rpm, en el combustible Ecopaís, considerando un aumento significativo del $2 \%$ en su Par motor con respecto al combustible Extra + Ferox. Con el aditivo Ferox, las dos mezclas de combustibles no tienen algún beneficio en el Torque del motor. Teniendo una disminución del 1\% al 2\% en su Par motor con respecto a los demás combustibles. 
Para un mejor análisis de los factores contaminantes es preciso trabajar con equipos calibrados y una buena condición de operación del motor de combustión interna (buen funcionamiento del convertidor catalítico, exceso de aire y una buena homogeneización de la mezcla aire- combustible), de tal manera se garantice el cumplimiento de las normas INEN.

\section{References}

[1] Yusri I. Alcohol based automotive fuels from first four alcohol family in compression and spark ignition engine. Renewable and Sustainable Energy Reviews. 2017;169.

[2] Ministerio de Hidrocarburos. EP Petroecuador firma contrato con empresas productoras de alcohol para garantizar abastecimiento de Ecopaís. Hidrocarburos. 2017 March. Obtenido de: www.hidrocarburos.gob.ec/ep-petroecuador-firma-contrato-c on-empresas-productoras-de-alcohol-para-garantizar-abastecimiento-de-ecopais/

[3] Saragosín LM. Incremento en la producción de etanol proveniente de la caña de azúcar. Fundamentos para disminuir la contaminación. 2016.

[4] Ferox. Ferox Fuel Tabs. Ferox. Obtenido de https://www.ferox.com.mx/wp-content/ uploads/As\{\%\}C3\{\%\}AD-funciona-FEROX.pdf

[5] Serpa G, Zumba X. Determinación de la influencia en emisiones contaminantes, torque, potencia y consumo de combustible de un vehículo con motor ciclo otto, de inyección electrónica de gasolina, mediante la variación de 6 tipos de bujías. Quito; 2016.

[6] Pardiñas J. Sistemas auxiliares del motor. España: Editex S.A; 2012.

[7] Faria M, Varella R, Duarte G, Farias T, Baptista P. Engine cold start analysis using naturalistic driving data: City level impacts on local pollutants emissions and energy consumption. Elsevier; 2018.

[8] Duarte, Goncalves, Farias. 2016.

[9] KIA 2013.

[10] Google Maps. 2018. Obtenido de https://www.google.com/maps/dir/ Supermaxi,+V\{\%\}C3\{\%\}ADa+Interoce\{\%\}C3\{\%\}A1nica+s\{\%\}2Fn+(C.C.+Villas+de+ Cumbay\{\%\}C3\{\%\}A1),+170157/-0.1787768,-78.4551195/-0.164806,-78.4535587/-0. 1647662,-78.468046/Rio+Coca+Y+de+Los+Shyris,+Quito/-0.1980181,-78.4866736/ $-0.1999809,-78.482$

[11] Quichimbla PFE, Solís SJM. Desarrollo de ciclos de conducción en ciudad, carretera y combinado para evaluar el rendimiento real del combustible de un vehículo con motor de ciclo otto en el distrito metropolitano de quito. Quito: Escuela Politécnica Nacional; 2017.

[12] Lopez T. 2013. 
[13] Pilliza, Montalvo. 2016.

[14] Espinoza H, Patino L, González Y, Irving L. Modelo predictivo de combustiÛn de mezclas de gas odelo predictivo de combustî̂n de mezclas de gas natural en motores de encendido por chispa (MECH). Ingeniería E Investigación. 2017;11-17.

[15] Recalde \& Revelo, 2015.

[16] Urbina. 2016.

[17] Caiza JPG, Portilla AÁA. Determinación de la Influencia de la Altura en emisiones contaminantes de un vehiculo con motor ciclo otto, de inyección electrónica de gasolina. Quito: Escuela Politecnica Nacional Master Thesis; 2010.

[18] Frey \& Eichenberger, 1997.

[19] Llanes Cedeño, Rocha-Hoyos, Peralta, \& Leguisamo, 2018.

[20] Araya A. Metodología de cálculo de emisiones vehiculares basada en modos de conducción y potencia específica vehícular. Santiago de Chile: Chile; 2008.

[21] Tolvett S. Análisis de emisiones de vehículos livianos según ciclos de conducción específicos para la región Metropolitana. Santiago de Chile: Chile; 2011.

[22] Dias de Carvalho R. Metodologia de cálculo de consumos de combustível e emissões de poluentes baseada em perfis de condução. Lisboa; 2014.

[23] Izquierdo, JFI, Campaña GGR, Andrés RR. Estudio de emisiones contaminantes producidas por un motor otto con el uso de gasolina y un combustible a base de 95\% de gasolina Y 5\% de etanol. Universidad Internacional del Ecuador; 2017.

[24] Kannan S, Saleh A, Nasir F. Review on bioethanol as alternative fuel for spark ignition engines. Renewable and Sustainable Energy Reviews. 2016;830-833.

[25] Dogan, Erol, Yaman, \& Kodanli, 2016.

[26] Rocha \& Zambrano 2015.

[27] Montero, JAC. Estudio de la combustión de un motor monocilíndrico de ignición alimentado con mezclas gasolina-etanol anhidro e hidratado a distintas concentraciones. 2011; 287-293.

[28] Frey C, Delavarrafiee M, Singh S. Real-world freeway and ramp activity and emissions for light-duty gasoline vehices. Transportion and Air Quality. 2014.

[29] Frey H, Unal A, Chen J, Li S. Modeling mobile source emissions based upon inuse and second-by-second data: Development of conceptual approaches for EPA's new MOVES model. Proceedings, Annual Meeting of the Air \& Waste Management Association, Pittsburgh, USA; 2003.

[30] Jiménez JL. Understanding and quantifying motor vehicle emissions with vehicle specific power and TILDAS remote sensing. Massachusetts Institute of Technology; 1999. 